\title{
Crystal growth and electrical transport properties of niobium and tantalum monopnictide and dipnictide semimetals
}

\author{
Hong Lu ${ }^{1}$, Shuang $\mathbf{J i a}^{\dagger 1,2}$ \\ ${ }^{1}$ International Center for Quantum Materials, School of Physics, Peking University, Beijing 100871, China \\ ${ }^{2}$ Collaborative Innovation Center of Quantum Matter, Beijing 100871, China \\ Corresponding author. E-mail: †'gwljiashuang@pku.edu.cn \\ Received March 13, 2017; accepted April 26, 2017
}

\begin{abstract}
The discovery of the first Weyl semimetal tantalum monoarsenide has greatly promoted physical research on the niobium and tantalum pnictide compounds. Crystallizing into the $\mathrm{NbAs}$ - and $\mathrm{OsGe}_{2}$-type structures, these mono- and di-pnictide semimetals manifest exotic electrical transport properties in magnetic field, which only occur in their single-crystalline forms. All the unusual electrical properties correspond to their poor carriers, which are indeed vulnerable to various crystal defects. In this review article, we present a comprehensive comparison of the crystal growth and electrical transport properties of the two semimetal families. We then discuss in detail the possible characteristic transport features, such as the chiral anomaly of Weyl quasiparticles. We emphasize the importance of crystal growth and sample manipulation for exploring the unique topological properties of Weyl semimetals in the future.
\end{abstract}

Keywords Weyl semimtal, crystal growth, electrical transport

PACS numbers 06.60.-c, 61.05.-a, 72.90.+y

Contents

1 Introduction

2 Crystal growth

3 Bulk and surface electron states

4 Transport properties

4.1 Magnetoresistance and Hall effect

4.1.1 $\mathrm{Nb}(\mathrm{Ta}) \mathrm{P}(\mathrm{As})$

4.1.2 $\mathrm{Nb}(\mathrm{Ta}) \mathrm{As}(\mathrm{Sb})_{2}$

4.2 Quantum oscillations

4.2.1 $\mathrm{Nb}(\mathrm{Ta}) \mathrm{P}(\mathrm{As})$

4.2.2 $\mathrm{Nb}(\mathrm{Ta}) \mathrm{As}(\mathrm{Sb})_{2}$

4.3 Negative longitudinal magnetoresistance

4.4 Measurements in extreme conditions

5 Discussion and outlook

Acknowledgements

References

\footnotetext{
*Special Topic: Recent Progress on Weyl Semimetals (Eds. Xincheng Xie, Xian-Gang Wan, Hong-Ming Weng \& Hua Jiang).
}

\section{Introduction}

Pnictogens (Pn), counting from phosphorus to bismuth, can react with almost every transition metal in the periodic table to form negatively charged pnictides. The moderate electro-negativity of the pnictogens places the transition-metal pnictides in an intermediate state between ionic and metallic solids [1]. While metallic states are commonly observed in transition-metal pnictides, there are semimetallic and semiconductive compounds as well. These semimetallic/semiconductive materials include the large groups of half-Heusler and skutterudite compounds, which have long been noticed as potentially useful materials in the electronic industry. In this review paper, we focus on two other small families of binary transition-element pnictide semimetals, the isostructural families of NbAs-type and $\mathrm{OsGe}_{2}$-type compounds, which have very recently come to the fore in condensed matter physics research.

Niobium monoarsenide (NbAs) crystallizes in a noncentrosymmetric, body-centered tetragonal structure 
(a)

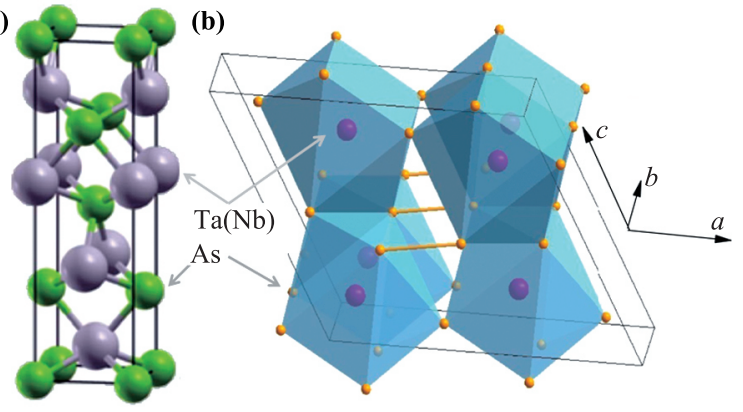

Fig. 1 Unit cells for $\mathrm{Ta}(\mathrm{Nb}) \mathrm{As}$ (a) and $\mathrm{Ta}(\mathrm{Nb}) \mathrm{As}_{2}$ (b).

that is rarely observed in transition metal monopnictides, compared with the ordinary hexagonal NiAs-type structure: only four compounds (NbP, NbAs, TaP, and TaAs) have been reported [Fig. 1(a)]. This structure was first reported in a centrosymmetric space group of $\mathrm{I} 4_{1} /$ amd for $\mathrm{Nb}(\mathrm{Ta}) \mathrm{P}$ in 1954 [2]. After a decade, the two arsenide siblings joined in and the structure for all four was corrected to a non-centrosymmetric group of $\mathrm{I} 4{ }_{1} \mathrm{md}$ [3-6]. This structure can be visualized as an arrangement of regular trigonal prisms of $\mathrm{Nb} / \mathrm{Ta}$ atoms with a $\mathrm{P} /$ As atom at the center [Fig. 1(a)] [7]. Four trigonal prisms per unit cell are stacked such that each prism is rotated and shifted with respect to its nearest neighbors [3]. The absence of a horizontal mirror plane in the unit cell makes this structure break the inversion symmetry, which is crucial to realize a time-reversal symmetric intact Weyl semimetal $[8,9]$. Their semimetal electronic states can be inferred by a simple ionic model in which the $\mathrm{Ta} / \mathrm{Nb}$ and $\mathrm{As} / \mathrm{P}$ atoms manifest $3^{+}$and $3^{-}$valence states in their coordinations, respectively [8]. The highest valence band arises for the $p^{6}$ electrons in $\mathrm{As}(\mathrm{P})$ and the $d^{3}$ electrons in $\mathrm{Nb}(\mathrm{Ta})$, while the lowest conduction band primarily consists of the $d$ electrons in $\mathrm{Nb}(\mathrm{Ta})$. The conduction and valence bands slightly intertwine, owing to the hybridization between the $d$ electrons of $\mathrm{Nb}(\mathrm{Ta})$ and $p$ electrons of $\operatorname{As}(\mathrm{P})$, leading to a semimetal state of $\mathrm{Nb}(\mathrm{Ta}) \operatorname{As}(\mathrm{P})$.

The transition metal dipnictides $\mathrm{MPn}_{2}(\mathrm{M}=\mathrm{V}, \mathrm{Nb}$, $\mathrm{Ta}, \mathrm{Cr}, \mathrm{Mo}$, and $\mathrm{W} ; \mathrm{Pn}=\mathrm{P}, \mathrm{As}$, and $\mathrm{Sb}$ ) crystallize in the monoclinic $\mathrm{OsGe}_{2}$-type structure [10-12]. The unit cell has one crystallographic $\mathrm{M}$ site and two Pn sites [Fig. 1(b)]. One of the two Pn sites is isolated from its nearby corresponding sites, while the other forms separated Pn-Pn dimers. Each $M$ atom is surrounded by six Pn atoms, which form a trigonal prism with two additional $\mathrm{Pn}$ atoms outside the rectangular faces. The prisms are stacked along the crystallographic $b$ direction through their trigonal faces to form the structure. Niobium and tantalum dipnictides are semimetallic as well. The Pn-Pn dimer has an electron number of $4^{-}$and the valences of the niobium and tantalum dipnictides can be written as $(\mathrm{Nb} / \mathrm{Ta})_{2}^{5+}(\mathrm{Pn}-\mathrm{Pn})^{4-} \mathrm{Pn}_{2}^{6-}[13,14]$, which leads to an electron-number balanced Zintl phase. Previous band-structure calculations [14] have confirmed their semimetal electronic states.

The physical properties of these two families of pnictides were rarely reported before 2015, especially for their single-crystalline forms in a magnetic field [15]. Previous measurements preformed on polycrystalline samples showed that NbAs and TaAs have metallic and semiconductive profiles of temperature-dependent resistivity $[\rho(T)]$, respectively, with a moderate positive magnetoresistance $\left[\mathrm{MR}=\left(\rho_{H}-\rho_{0}\right) / \rho_{0}=1-3\right]$ in $8 \mathrm{~T}$ at $2 \mathrm{~K}$ [16]. Thermoelectric measurements revealed two types of carriers coexistent in NbAs and TaAs. Polycrystalline $\mathrm{V}(\mathrm{Nb}, \mathrm{Ta}) \mathrm{Sb}_{2}$ were also reported to have a metallic profile of $\rho(T)$ with a moderate $\mathrm{MR}=1-2$ at the base temperature in $12 \mathrm{~T}$ [17]. These sporadic transport measurements of polycrystals only limitedly demonstrated their intrinsic electronic states, because the impurities at the grain boundaries strongly affect the scattering processes. On the other hand, the transport properties of a single crystal are much more spectacular. A debut work on single-crystalline $\mathrm{NbSb}_{2}$ grown by the flux method in 2014 described a large, parabolic field-dependent MR up to 1300 at $2 \mathrm{~K}$ in $9 \mathrm{~T}$ [17].

In 2015, angle-resolved photoemission spectroscopy (ARPES) experiments revealed TaAs as the first inversion-symmetry-breaking Weyl semimetal $[8,9,18$, 19]. Thenceforth, the number of investigations of the single crystals of $\mathrm{Nb}(\mathrm{Ta}) \mathrm{As}(\mathrm{P})$ and $\mathrm{Nb}(\mathrm{Ta}) \mathrm{As}(\mathrm{Sb})_{2}$ compounds has increased dramatically. Later, ARPES experiments on NbAs and TaP confirmed the existence of Weyl quasiparticles as their low-energy exciton states [20-23]. Alongside the ARPES results, the magnetotransport properties of the single crystals of NbAstype compounds featured strong quantum oscillations (QOs), extremely high mobility, and large transversal $\mathrm{MR}$, as well as a negative longitudinal magnetoresistance (LMR) [24-27]. Weyl quasiparticles in the bulk and Fermi arc surface states of the NbAs-type semimetals motivated researchers to test multi-aspect conceptions in Weyl physics, including quantum interference on the surface state [28-30] and the optical response of the chiral anomaly [31]. Besides the research on $\mathrm{Nb}(\mathrm{Ta}) \mathrm{As}(\mathrm{P})$ compounds, the magneto-transport properties of the single-crystalline $\mathrm{Nb}(\mathrm{Ta}) \mathrm{As}(\mathrm{Sb})_{2}$ compounds were also checked exclusively. These compounds also exhibit extremely large MR and high carrier mobilities [32-36], but band structure calculations revealed that they are weakly topological semimetals, rather than Weyl semimetals. All these developments give us insight to recognize the topological aspect of the physical properties of transition-element pnictide semimetals.

Generally speaking, the topological identity of the 


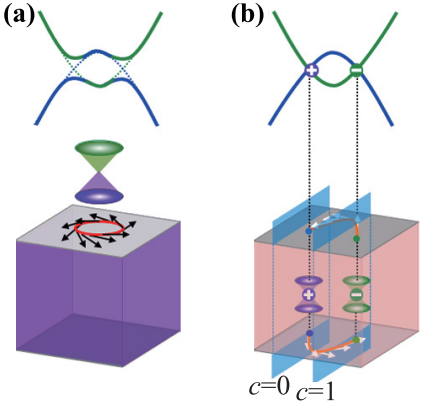

Fig. 2 Schematics of a TI (a) and Weyl semimetal (b) (Reproduced from Ref. [37]). (a) A TI has a bulk band gap with a band inversion. Its surface states exhibit a Dirac-conelike dispersion. (b) A Weyl semimetal is gapless in the bulk with pairs of band crossing points. The Chern number is not zero between pairs of Weyl points with opposite chirality, which leads to a surface Fermi arc.

semimetals stems from the topological insulators (TIs). TIs differ from ordinary band insulators owing to their characteristic topological invariants of the electrical structures [38]. TIs present an electrically insulating bulk state but a metallic surface state with linear energy dispersion, in which the crossing point can be well described by a two-dimensional (2D) Dirac equation [Fig. 2(a)]. These topological concepts can be broadened to the topological semimetals, where the conduction and valence bands only cross at isolated points that can be viewed as topological defects. As the first realized cataloged topological semimetals, Dirac semimetals, including $\mathrm{Na}_{3} \mathrm{Bi}$ and $\mathrm{Cd}_{3} \mathrm{As}_{2}$ [39, 40], host the band crossing points with the linear energy dispersion relation, which can be viewed as three-dimensional (3D) analogies of the topological surface state of TIs. A Weyl semimetal is similar to a Dirac semimetal, but its band crossing points, called Weyl nodes, are single degenerate and spin-momentum locked. The Weyl nodes with definite chirality can be described by a Weyl equation, which is well-known from the standard model in quantum field theory.

The discovery of the Weyl semimetals has important significance in both fundamental and applied physics. It first paves the way to test important concepts in highenergy physics such as the famous chiral anomaly in table-top experiments. The chiral charge of each Weyl node can be understood as a magnetic monopole or an anti-monopole in momentum space. Parallel magnetic and electric fields can pump electrons between Weyl nodes of opposite chirality that are separated in momentum space. This process violates the conservation of the chiral charge and gives rise to an analogue of the chiral anomaly in condensed matter. Secondly, the Fermi arc surface state would violate quantum mechanics in any pure $2 \mathrm{D}$ electron system, but is allowed on the surface of a Weyl semimetal, owing to the existence of Weyl nodes. However, finding a Weyl semimetal has been a challenging task because one must break time-reversal or space-inversion symmetry to realize Weyl fermions in a solid. Several compounds, such as pyrochlore iridate $\mathrm{Y}_{2} \mathrm{Ir}_{2} \mathrm{O}_{7}$ [41] and spinel $\mathrm{HgCr}_{2} \mathrm{Se}_{4}$ [42], have been predicted as Weyl semimetals, but no candidate has been experimentally confirmed until the discovery of the first Weyl semimetal, TaAs.

Many exotic electrical transport properties have been predicted for the Weyl quasiparticles in Weyl semimetals (see Ref. [43] and the references therein). These crucial features of Weyl semimetals have motivated tremendous endeavors in the study of $\mathrm{Nb}(\mathrm{Ta}) \mathrm{P}(\mathrm{As})$ compounds, aiming for their potential applications in electronics. In this review, we summarize the results of the recently reported transport properties of NbAs-type and $\mathrm{OsGe}_{2}-$ type compounds. Crystal growth and electronic structure are briefly introduced before the survey of the transport experiments. We then present the transport data in four aspects: the classical properties including MR, carrier densities, and mobilities; the quantum oscillations (QOs); the negative longitudinal magnetoresistance (LMR), which was debatably attributed to the chiral anomaly; and the properties under extreme conditions such as high pressure and intense magnetic field. After the survey, we discuss in detail the comparison of the observed and the allegedly characteristic transport features originating from the Weyl quasiparticles.

\section{Crystal growth}

Polycrystals of the $\mathrm{Nb}(\mathrm{Ta}) \mathrm{P}(\mathrm{As})$ and $\mathrm{Nb}(\mathrm{Ta}) \mathrm{As}(\mathrm{Sb})_{2}$ compounds can be easily obtained by reacting the mixtures of stoichiometric $\mathrm{Nb}(\mathrm{Ta})$ powders and red phosphorus, arsenic, or antimony powders in evacuated fusedsilica ampules at high temperatures $[2,5]$. Single crystals of $\mathrm{Nb}(\mathrm{Ta}) \mathrm{As}$ and $\mathrm{Nb}(\mathrm{Ta}) \mathrm{As}_{2}$ were obtained as early as the 1960s via the chemical vapor transfer (CVT) method with polycrystals as the sources and halogen elements as the agents $[5-7,12,44]$. Most of the recent crystal growth experiments followed these CVT processes and optimized the conditions via selecting different transfer agents, temperature gradients and extending the growth time. The adequate transfer agents include $\mathrm{TaBr}_{5}$ for the growth of TaAs and $\mathrm{TaAs}_{2}$, and $\mathrm{NbI}_{5}$ for the growth of $\mathrm{NbAs}_{2}[24,25]$. It is noteworthy that the temperature gradients for the growth of $\mathrm{Nb}(\mathrm{Ta}) \mathrm{P}(\mathrm{As})$ were reported to be positive (from the hot source to the cold sink) $[26,45]$ or negative (from the cold source to the hot sink) in different literatures [46, 47]. Recent work by Li et al. focused on this discrepancy for the growth of TaAs [48]. They reported a CVT growth for largesize TaAs single crystals using iodine as the agent. The 
(a)

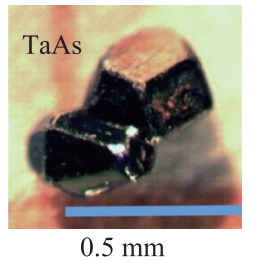

(b)

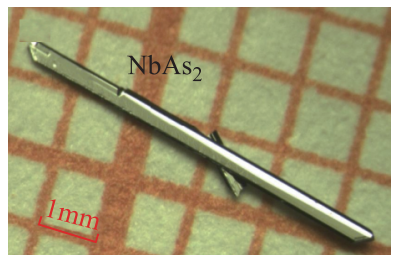

Fig. 3 Single crystals of TaAs and $\mathrm{NbAs}_{2}$ (Reproduced from Refs. [8] and [35]).

reaction between the solid and the agent:

$$
\operatorname{TaAs}(\mathrm{s})+4 \mathrm{I}_{2}(\mathrm{~g}) \longleftrightarrow \mathrm{TaI}_{5}(\mathrm{~g})+\mathrm{AsI}_{3}(\mathrm{~g})
$$

is exothermic. The TaAs crystals form at the hot end when the gaseous $\mathrm{TaI}_{5}$ and $\mathrm{AsI}_{3}$ absorb the heat and migrate from the cold end. A general description of the CVT growth can be found in Ref. [49].

The growth of single-crystalline $\mathrm{Nb}(\mathrm{Ta}) \mathrm{P}(\mathrm{As})$ and $\mathrm{Nb}(\mathrm{Ta}) \mathrm{P}(\mathrm{As}, \mathrm{Sb})_{2}$ from the melt has never been attempted because of their high liquidus temperatures, as well as highly versatile and toxic phosphorus and arsenic compositions. The flux method was employed instead for successfully growing crystals at relatively low temperature [50]. Self-flux growth [32] was only reported for single crystals of $\mathrm{NbSb}_{2}$. These crystals behave similarly in the transport measurement as those of $\mathrm{NbAs}_{2}$ and $\mathrm{TaAs}_{2}$ grown via the CVT method. Low-melting-point elements such as gallium and tin have been used as the flux to grow TaAs and NbAs crystals $[30,51]$, but their physical properties are rarely reported, probably owing to their small size.

The morphologies of the single crystals of particular structures grown via different methods are similar in general. The $\mathrm{Nb}(\mathrm{Ta}) \mathrm{P}(\mathrm{As})$ single crystals are shaped like a millimeter-sized edge-cut octahedral or small square plate [Fig. 3(a)]. The square or rectangle surfaces corresponding to the crystallographic $c$ plane are easy to recognize (Fig. 3). The single crystals of $\mathrm{Nb}(\mathrm{Ta}) \mathrm{P}(\mathrm{As}$, $\mathrm{Sb})_{2}$ have a blade-like shape with an approximate length of several millimeters and a width less than $1 \mathrm{~mm}$ [Fig. 3(b)]. The long direction of the blade-like crystals is the $b$ direction, while the flat plane is perpendicular to the (001) direction.

Similar to the case of semiconductors, the defects in semimetals can also significantly alter their electrical transport properties. Multiple types of defects were realized early in $\mathrm{Nb}(\mathrm{Ta}) \mathrm{P}(\mathrm{As})$, but their effects on the physical properties were only examined very recently. These defects include, but are not limited to, non-stoichiometry and stacking disorders. X-ray diffraction (XRD) and energy dispersive spectroscopy (EDS) revealed phosphorous deficiency in $\mathrm{NbP}$ and $\mathrm{TaP}$, but tantalum deficiency in TaAs $[2,52]$. Stacking disorder is a particular type of defect observed in the non-centrosymmetric $\mathrm{Nb}(\mathrm{Ta}) \mathrm{P}(\mathrm{As})$ family. Previous studies suggested that

a metastable Tungsten Carbonide (WC)-type structure forms during the early stages of the synthesis reaction for these monopnictides [53]. The residues at room temperature (RT) remain the metastable WC-type structure parts, which are sandwiched between the tetragonal parts stacking along the $c$ axis $[3,53]$. Recent measurements observed a high density of stacking faults in TaP and TaAs single crystals [52]. Notwithstanding the high density of defects, which presumably affect the electrical structure according to the calculations, single-crystalline TaP has still experimentally shown strong QOs and a high carrier mobility [52]. The correlation between the defects and transport properties has not been addressed, but an investigation on TaAs crystals grown in different conditions showed that the crystals have distinct transport properties owing to the different chemical potentials induced by their defects [27]. Strong sample dependence of the transport properties was also observed in $\mathrm{NbAs}_{2}$ and $\mathrm{TaAs}_{2}$ [35], but the origin and effects of the defects have not been addressed.

\section{Bulk and surface electron states}

A brief description of the bulk and surface electron structures of $\mathrm{NbAs}$-type and $\mathrm{OsGe}_{2}$-type compounds can help to better understand their electrical properties. Determination of the electron structures primarily depends on band structure calculations and ARPES experiments. First-principle calculations suggest that the conduction and valence bands of both families cross each other along the high-symmetry trajectory in their Brillouin zone (BZ) without spin-orbital coupling (SOC). For NbAstype compounds, these band crossings take the form of a closed curve projected as nodal lines on the mirror planes of the BZ in the $x$ and $y$ directions [Fig. 4(a)]. In the presence of SOC, the band structure is fully gapped along all the high-symmetry directions, and each line
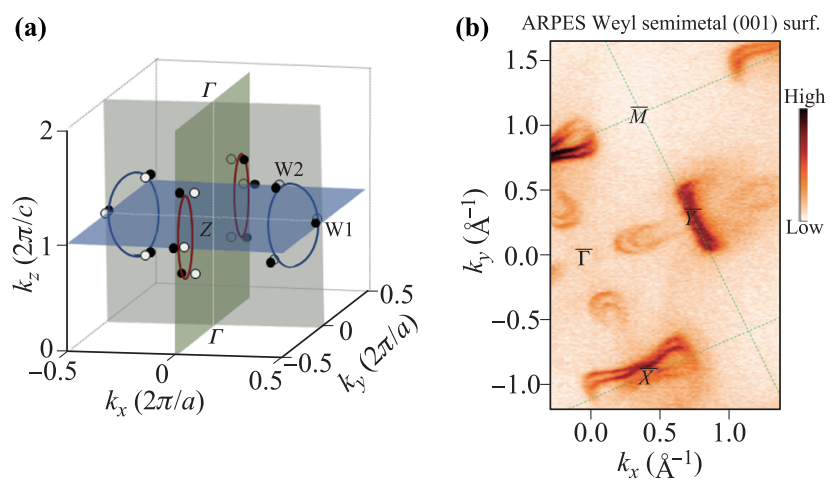

Fig. 4 (a) Location of the Weyl nodes in the Brillouin zone for NbAs-type compounds (Reproduced from Ref. [8]). (b) The ARPES-measured Fermi surface of the (001) cleaving plane of TaAs (Reproduced from Ref. [18]). 
node vaporizes into six Weyl points shifted slightly away from the mirror planes. There are 24 Weyl nodes in total: eight Weyl points on the $k_{z}=2 \pi / c$ plane, which are called $\mathrm{W} 1$, and 16 Weyl points away from the plane, which are called W2. It is noteworthy that the notations of $\mathrm{W} 1$ and $\mathrm{W} 2$ are opposite in different literatures $[8,9]$, and we choose the notation in Ref. [8] in this paper. The two types of Weyl nodes are located at slightly different energies, which vary from 20 to $50 \mathrm{meV}$ for different $\mathrm{Nb}(\mathrm{Ta}) \mathrm{P}(\mathrm{As})$ compounds $[8,9,37,54]$.

The existence of Weyl nodes in $\mathrm{Nb}(\mathrm{Ta}) \mathrm{P}(\mathrm{As})$ promises a new type of robust, unclosed Fermi surface on the interface between the bulk and the vacuum. Each Weyl node behaves as a source or sink of the Berry flux in momentum space. As a consequence of this monopole feature, the projections of the Weyl nodes with opposite chirality near the surfaces of BZ boundaries must be connected by surface Fermi arcs. Figure 4(b) shows the surface state observed by ARPES experiments on the (001) plane of TaAs, which is characterized by a crescent-like feature consisting of two curves that join each other at the two end points. The two curves of the crescent are two Fermi arcs, and the two end points correspond to the projected W2. Both the Weyl fermions in the bulk and the Fermi arcs on the surface of $\mathrm{Nb}(\mathrm{Ta}) \mathrm{P}(\mathrm{As})$ can be selectively probed in ARPES experiments by choosing different incident photon energies [18-21, 23, 55].

In contrast, all the nodal lines in $\mathrm{Nb}(\mathrm{Ta}) \mathrm{Pn}_{2}$ become fully gapped in the presence of SOC, which leads to completely separated electron and hole bands [56]. The calculation also shows that $\mathrm{Nb}(\mathrm{Ta}) \mathrm{As}_{2}$ and $\mathrm{NbSb}_{2}$ are close to the compensated semimetals with equal elec- tron and hole densities. Both the electron and hole bands are weakly topological, leading to a fragile surface state [57].

\section{Transport properties}

The electrical transport experiments in a magnetic field give the fundamental properties of the carriers in $\mathrm{Nb}(\mathrm{Ta}) \mathrm{P}(\mathrm{As})$ and $\mathrm{Nb}(\mathrm{Ta}) \mathrm{As}(\mathrm{Sb})_{2}$ compounds. In this part, we summarize the transport properties of the single crystals in four aspects: the classical properties including MR, carrier densities and mobilities; the electrical properties associated with the Shubnikov-de Haas $(\mathrm{SdH})$ and de Haas-van Alphen (dHvA) oscillations; the negative LMR; and the properties under high pressure and strong magnetic field.

\subsection{Magnetoresistance and Hall effect}

\subsection{1 $\mathrm{Nb}(\mathrm{Ta}) \mathrm{P}(\mathrm{As})$}

Since the as-grown crystals of $\mathrm{Nb}(\mathrm{Ta}) \mathrm{P}(\mathrm{As})$ have large plates of (001) surface [Fig. 3(a)] in general, making bar-shape samples with the current along the $a$ direction $(i / / a)$ is convenient for transport measurements. The measurements of the current along the (110) and (001) directions $[27,63]$ of the samples show similar features. The temperature-dependent resistivity of all the crystals of $\mathrm{Nb}(\mathrm{Ta}) \mathrm{P}(\mathrm{As})$ show a metallic profile in zero magnetic field. The residue resistivity ratio defined as $R R R=R(300 \mathrm{~K}) / R(2 \mathrm{~K})$ varies from 40 to 100 for dif-

Table 1 Summary of the basic magneto-transport properties for single-crystalline NbAs-type compounds. The properties include RRR and MR at high and low temperatures. The carrier densities of electron $(n)$ and hole $(p)$ and mobilities $\left(\mu_{n}\right.$ and $\left.\mu_{p}\right)$ at the base temperature $(\sim 2 \mathrm{~K})$ were estimated by a two-band model $(\mathrm{T})$ or a single-band model (S).

\begin{tabular}{|c|c|c|c|c|c|c|}
\hline Compound & RRR & MR & $n(p) /\left(10^{19} \mathrm{~cm}^{-3}\right)$ & $\mu_{n}\left(\mu_{p}\right) /\left(10^{4} \mathrm{~cm}^{2} \cdot \mathrm{V}^{-1} \cdot \mathrm{s}^{-1}\right)$ & Note & Ref \\
\hline \multirow[t]{3}{*}{$\mathrm{NbP}$} & 115 & $\begin{array}{c}2.5_{\mathrm{RT}, 9 \mathrm{~T}}, 8500_{1.85 \mathrm{~K}, 9 \mathrm{~T}} \\
3.6 \times 10_{1.3 \mathrm{~K}, 30 \mathrm{~T}}^{4}, 8.1 \times 10_{1.5 \mathrm{~K}, 60 \mathrm{~T}}^{4}\end{array}$ & 0.15 & 500 & $\mathrm{~S}$ & {$[25]$} \\
\hline & - & - & $0.942(n), 0.924(p)$ & - & $\mathrm{T}$ & {$[58]$} \\
\hline & $25-100$ & $1 \times 10_{2 \mathrm{~K}, 15 \mathrm{~T}}^{4}$ & 0.2 & $500-1000$ & $\mathrm{~S}$ & {$[59]$} \\
\hline \multirow{2}{*}{$\mathrm{TaP}$} & $\sim 100$ & $180_{2 \mathrm{~K}, 9 \mathrm{~T}}$ & $1.2(n), 0.6(p)$ & $2(\mathrm{e}), 9(\mathrm{~h})$ & $\mathrm{T}$ & {$[47]$} \\
\hline & 96 & $160_{200 \mathrm{~K}, 56 \mathrm{~T}}, 1.8 \times 10_{2 \mathrm{~K}, 9 \mathrm{~T}}^{4}, 1.4 \times 10_{1.5 \mathrm{~K}, 56 \mathrm{~T}}^{5}$ & - & $20(\mathrm{e}), 40(\mathrm{~h})$ & $\mathrm{T}$ & {$[46]$} \\
\hline \multirow[t]{2}{*}{$\mathrm{NbAs}$} & 72 & $2_{\mathrm{RT}, 9 \mathrm{~T}}, 2300_{2 \mathrm{~K}, 9 \mathrm{~T}}$ & 1.8 & 35 & $\mathrm{~S}$ & {$[61]$} \\
\hline & 72 & $4620_{1.9 \mathrm{~K}, 18 \mathrm{~T}}$ & $1.08(n), 0.904(p)$ & $19(\mathrm{e}), 190(\mathrm{~h})$ & $\mathrm{T}$ & {$[62]$} \\
\hline
\end{tabular}


ferent samples (Table 1). Figures 5(a) and (b) show the representative $\rho(T)$ curves in different magnetic fields and the MR versus field at different temperatures for TaP, respectively. Their MR is commonly reported in the range of $10^{3}-10^{4}$ at $2 \mathrm{~K}$ and $1-3$ at $\mathrm{RT}$ in $9 \mathrm{~T}$, which is extremely large compared with regular metals.

The power law of the MR for $\mathrm{Nb}(\mathrm{Ta}) \mathrm{P}(\mathrm{As})$ is strongly dependent on temperature, magnetic field, and the samples. Most of the experiments reported a quadratic fielddependent MR in low magnetic field, which is consistent with the depiction by a two-band model. This quadratic power relation evolves to linear in some samples when the magnetic field increases at low temperatures, with a crossover behavior in the range 1-10 $\mathrm{T}$ [Fig. 5(b)]. The linear, unsaturated MR at low temperatures was reported to persist up to $55 \mathrm{~T}$ at low temperatures for

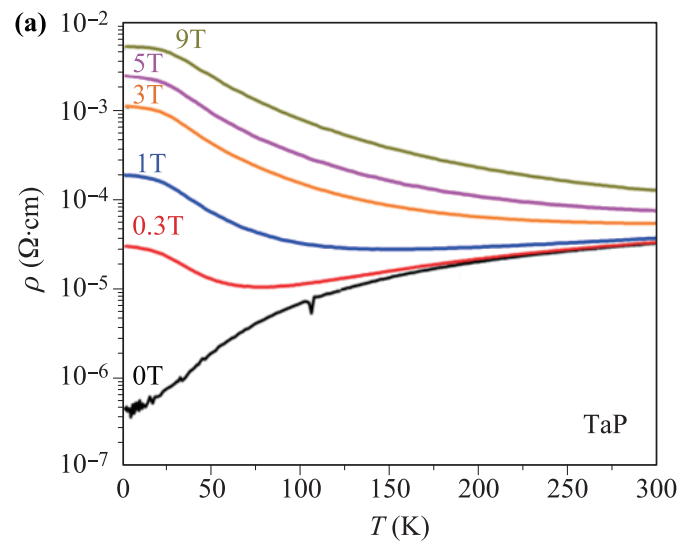

$\mathrm{TaP}$ and TaAs [24, 64]. However, other works in the literatures reported that the MR of different samples of $\mathrm{TaP}$ and $\mathrm{NbP}$ tends to saturate in high magnetic field $[45,59]$. It is noteworthy that the $\mathrm{SdH}$ oscillations for some samples of the $\mathrm{Nb}(\mathrm{Ta}) \mathrm{P}(\mathrm{As})$ compounds can be strongly amplified in high field at low temperatures, which makes the power law difficult to determine. At high temperatures, all the literatures reported that the MR significantly drops while the power law changes to near-quadratic in the whole field range [Fig. 5(b)].

Hall measurements provide more information for the electron- and hole-type carriers. Figure 6(a) shows the Hall resistivity $\left(\rho_{y x}\right)$ with respect to the field at different temperatures for a single crystal of TaP. The profiles at different temperatures are typical for all the $\mathrm{Nb}(\mathrm{Ta}) \mathrm{P}(\mathrm{As})$ compounds in the literatures $[26,46]$. The

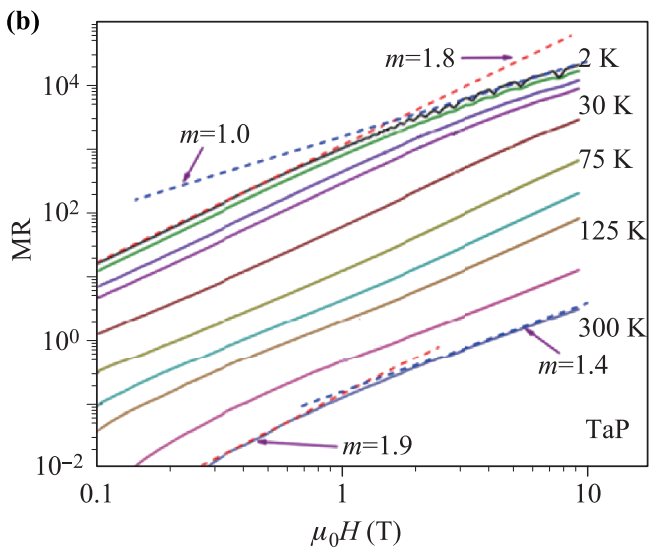

Fig. 5 Resistivity for single crystals of TaP in magnetic fields (Reproduced from Ref. [46]). (a) Temperature-dependent resistivity in different magnetic fields for TaP. (b) MR versus the magnetic field on a double-log scale for TaP at different temperatures. The power law for the MR at low temperatures changes from $\mathrm{MR} \propto H^{1.8}$ in low field to MR $\propto H$ in high field.
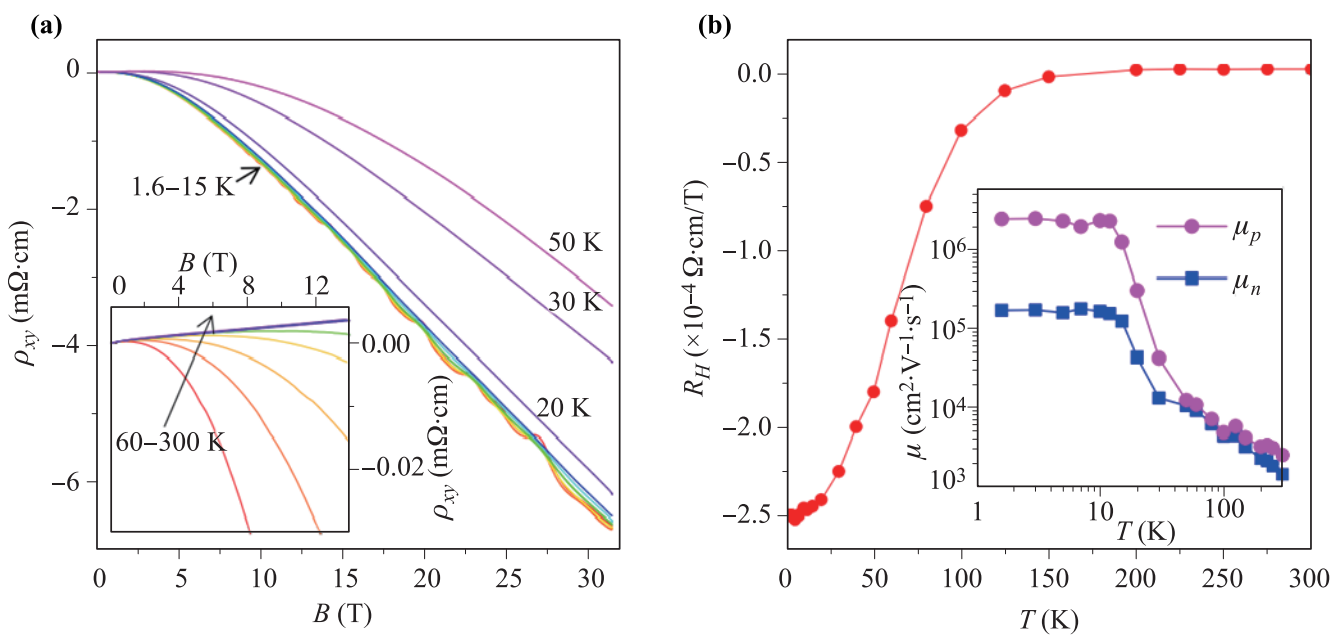

Fig. 6 Hall effect for a single crystal of TaP (Reproduced from Ref. [45]). (a) Hall resistivity versus field at different temperatures from 1.6 to $50 \mathrm{~K}$. Inset: From $60 \mathrm{~K}$ to $300 \mathrm{~K}$. (b) Hall coefficient $\left(R_{H}\right)$ at different temperatures. $R_{H}$ was obtained from the fitting based on a single-band model (see details in text). Inset: The values of $\mu_{n}$ and $\mu_{p}$ were estimated from the fitting based on a two-band model. 
Hall resistivity is positive and linearly field-dependent up to $9 \mathrm{~T}$ at high temperatures, indicating $p$-type carriers' contribution. When the temperature drops, $\rho_{y x}$ continuously bends down to be negative in higher magnetic field. At the base temperature, $\rho_{y x}$ shows a flat nonlinear part in low field, and a negative linear part in high field, which is imposed by strong $\mathrm{SdH}$ oscillations.

There are two models used to analyze the Hall data to obtain information of the carrier densities and mobilities. A single-band Drude model is used to calculate the carrier densities by $n, p=1 /\left[e R_{H}\right]$ and the mobilities by $\mu_{e, h}=R_{H} / \rho_{x x}$, where $R_{H}$ is the Hall coefficient equal to the slope of the linearly dependent $\rho_{y x}(H)$. This model adapts well for the $\rho_{y x}(H)$ at high temperatures. For the nonlinear $\rho_{y x}(H)$ at low temperatures, the slope of $\rho_{y x}(H)$ in high field is selected to calculate $R_{H}$. In order to obtain information of the other type of carriers, a two-band Drude model is used to fit the nonlinear curves of $\rho_{y x}(H)$ and estimate the densities and mobilities for both electrons and holes. Here, the data analysis is more complicated: the Hall conductivity tensor $\sigma_{x y}=\rho_{y x} /\left(\rho_{x x}^{2}+\rho_{y x}^{2}\right)$ is first calculated and then fitted by adopting [65]

$$
\sigma_{x y}=\left[n_{h} \mu_{h}^{2} \frac{1}{1+\left(\mu_{h} H\right)^{2}}-n_{e} \mu_{e}^{2} \frac{1}{1+\left(\mu_{e} H\right)^{2}}\right] e H .
$$

The four fitted parameters above carry large uncertainties. As shown in Table 1, the fittings by the single-band model (labeled as S) in general estimate much greater mobilities than those by the two-band model (labeled as $\mathrm{T})$. Comparable fitting results for some samples of $\mathrm{NbAs}$ can be found in Refs. [61] and [62], where the single-band model estimated the mobility 100 times larger than that by the two-band model. Nevertheless, all the analyses yield the mobility more than $10^{5} \mathrm{~cm}^{2} /(\mathrm{V} \cdot \mathrm{s})$ at the base temperature, while the carrier densities of electrons and holes are in the range of $10^{18}-10^{19} \mathrm{~cm}^{-3}$.

\subsection{2 $\mathrm{Nb}(\mathrm{Ta}) \mathrm{As}(\mathrm{Sb})_{2}$}

The $\rho(T)$ curves in different magnetic fields and the $\mathrm{MR}(H)$ curves at different temperatures for a single crystal of $\mathrm{NbAs}_{2}$ are shown in Figs. 7(a) and (b), respectively. These profiles have been commonly reported for the sibling compounds in the literature (Table 2). Generally, the $\mathrm{MR}$ of $\mathrm{Ta}(\mathrm{Nb}) \mathrm{As}(\mathrm{Sb})_{2}$ is as large as that of $\mathrm{Nb}(\mathrm{Ta}) \mathrm{P}(\mathrm{As})$ at low temperatures but one order of magnitude lower at high temperatures. This big difference in MR between high and low temperatures manifests a field-induced transition-like behavior in the $\rho(T)$ curves occurring at around $100 \mathrm{~K}$, similar to what was also observed for the semimetal $\mathrm{WTe}_{2}[67]$. The $\mathrm{SdH}$ oscillations are pronounced below $5 \mathrm{~K}$ on the background of the parabolic field-dependent resistance for the $\mathrm{Ta}(\mathrm{Nb}) \mathrm{As}(\mathrm{Sb})_{2}$ family. All the $\operatorname{MR}(H)$ curves of the $\mathrm{Nb}(\mathrm{Ta}) \mathrm{As}(\mathrm{Sb})_{2}$ compounds show a power law very close to parabolic at high and low temperatures.

Hall measurements revealed two types of carriers in $\mathrm{Nb}(\mathrm{Ta}) \mathrm{As}(\mathrm{Sb})_{2}$ as well. The carrier densities and mobilities obtained by the two-band model fitting are summarized in Table 2. These measurements confirmed the compensated semimetal states predicted by the band structure calculations with $n$ and $p$ on the order of $10^{19} \mathrm{~cm}^{-3}$ at the base temperature [56]. The mobilities were estimated on the order of $10^{4} \mathrm{~cm}^{2} /(\mathrm{V} \cdot \mathrm{s})$, which are in general one order of magnitude less than those of $\mathrm{Nb}(\mathrm{Ta}) \mathrm{P}(\mathrm{As})$.

\subsection{Quantum oscillations}

$\mathrm{SdH}$ and dHvA effects present the oscillations in a material's electrical resistance and magnetization in a mag-

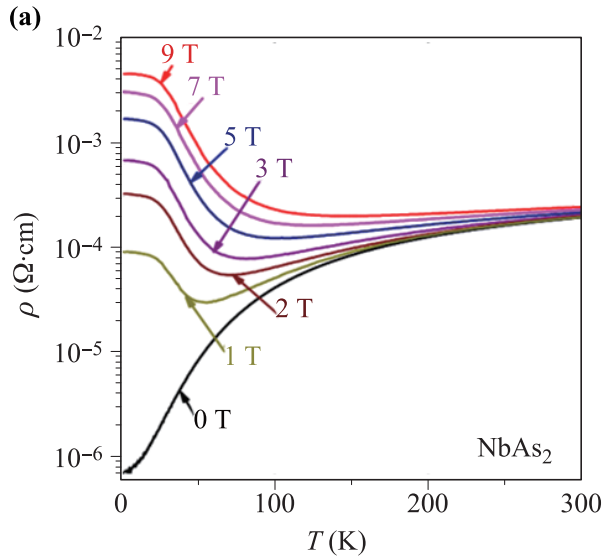

(b)

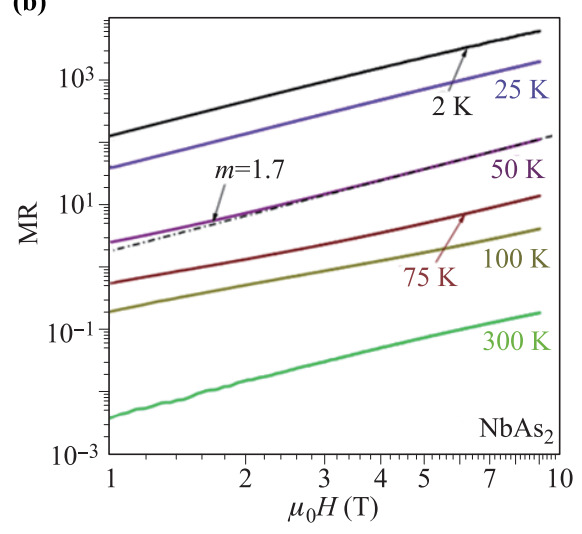

Fig. 7 Resistivity for the single crystals of $\mathrm{NbAs}_{2}$ in magnetic fields (Reproduced from Ref. [35]). (a) Temperaturedependent resistivity in different magnetic fields for $\mathrm{NbAs}_{2}$. (b) MR versus magnetic field on a double-log scale for NbAs 2 at different temperatures. $\mathrm{MR} \propto H^{2}$ at all temperatures. 
Table 2 Summary of the basic electrical transport properties of the single crystals of $\mathrm{Nb}(\mathrm{Ta}) \mathrm{As}(\mathrm{Sb})_{2}$. The properties include RRR and MR at high and low temperatures. The carrier densities of electron $(n)$ and hole $(p)$ and mobilities $\left(\mu_{n}\right.$ and $\left.\mu_{p}\right)$ at the base temperature $(\sim 2 \mathrm{~K})$ were estimated by a two-band model $(\mathrm{T})$ or a single-band model (S).

\begin{tabular}{|c|c|c|c|c|c|c|}
\hline Compound & RRR & MR & $n(p) /\left(10^{19} \mathrm{~cm}^{-3}\right)$ & $\mu_{n}\left(\mu_{p}\right) /\left(10^{4} \mathrm{~cm}^{2} \cdot \mathrm{V}^{-1} \cdot \mathrm{s}^{-1}\right)$ & Note & Ref. \\
\hline \multirow[t]{2}{*}{$\mathrm{NbAs}_{2}$} & 317 & $8800_{2 \mathrm{~K}, 9 \mathrm{~T}}$ & 2 & 10 & $\mathrm{~T}$ & {$[35]$} \\
\hline & 75 & $1000_{2.5 \mathrm{~K}, 14 \mathrm{~T}}$ & - & 10 & $\mathrm{~T}$ & {$[66]$} \\
\hline \multirow[t]{3}{*}{$\mathrm{TaAs}_{2}$} & 1000 & $0.12_{\mathrm{RT}, 9 \mathrm{~T}}, 12000_{2 \mathrm{~K}, 9 \mathrm{~T}}$ & $0.28(n), 0.28(p)$ & $12(\mathrm{e}), 12(\mathrm{~h})$ & $\mathrm{T}$ & {$[33]$} \\
\hline & 83 & $7300_{2.5 \mathrm{~K}, 14 \mathrm{~T}}$ & - & 10 & $\mathrm{~T}$ & {$[66]$} \\
\hline & 100 & $2000_{0.5 \mathrm{~K}, 9 \mathrm{~T}}, 40000_{0.5 \mathrm{~K}, 65 \mathrm{~T}}$ & $1.4(\mathrm{e}), 1.0(\mathrm{~h})$ & $0.19(\mathrm{e}), 0.25(\mathrm{~h})$ & $\mathrm{T}$ & {$[57]$} \\
\hline $\mathrm{NbSb}_{2}$ & 450 & $1300_{2 \mathrm{~K}, 9 \mathrm{~T}}, 43000_{0.4 \mathrm{~K}, 32 \mathrm{~T}}$ & - & - & - & {$[32]$} \\
\hline
\end{tabular}

netic field, respectively. These two QO effects are powerful tools for probing the electronic structure of materials [68]. When an external magnetic field is applied to a metallic crystal, the energy of the conduction electrons is quantized by the applied field into a series of Landau subbands. As the magnetic field increases, the Landau subbands successively pass through the Fermi level $\left(E_{F}\right)$ while such passings occur at equal intervals of $1 / H$ given by

$$
\Delta(1 / H)=2 \pi e /\left(\hbar A_{F}\right),
$$

where $A_{F}$ is the extremal cross-sectional area of the Fermi surface (FS) by planes normal to $H$; $e$ is the electron charge and $h$ is Planck's constant $[\hbar=h /(2 \pi)]$. The oscillation frequency $F$, defined as $1 / \Delta(1 / H)$, is given by $F=\frac{\hbar}{2 \pi e} A_{F}$. With an increasing magnetic field being applied, the fluctuations of the density of states (DOS) at $E_{F}$ result in the oscillations of the magnetization in the dHvA effect. On the other hand, because the probability of the scattering in electrical transport is also proportional to the DOS, the resistance oscillates with increasing magnetic field in the $\mathrm{SdH}$ effect.

Recent magneto-transport and torque measurements observed the $\mathrm{SdH}$ and $\mathrm{dHvA}$ oscillations in all the $\mathrm{Nb}(\mathrm{Ta}) \mathrm{As}(\mathrm{Sb})_{2}$ and $\mathrm{Nb}(\mathrm{Ta}) \mathrm{P}(\mathrm{As})$ compounds. The analyses of these QOs can precisely map out the FS topology and determine the exact $E_{F}$ value by comparing with the band structure calculations, which cannot be accessed using the regular Hall effect measurements. QO measurements have the ability to track $E_{F}$ within the range of a few milli-electron volts, which is beyond that of ARPES measurements. Because the chirality of the electron pockets is only well-defined within a few millielectron volts near the Weyl points in $\mathrm{Nb}(\mathrm{Ta}) \mathrm{As}(\mathrm{P})$, pre- cisely determining the position of $E_{F}$ is crucial to understand anomalous transport phenomena. Furthermore, the analyses of the phase shift of $\mathrm{SdH}$ and dHvA oscillations have been used to determine the Berry phase of the electron pockets [69-72], which is an important testimony to their non-trivial topology.

\subsection{1 $\mathrm{Nb}(\mathrm{Ta}) \mathrm{P}(\mathrm{As})$}

The detected QO frequencies for different crystals of $\mathrm{Nb}(\mathrm{Ta}) \mathrm{P}(\mathrm{As})$ when the magnetic field is along the crystallographic $c$ and $a$ directions are summarized in Table 3. Comparing the measured QOs and the calculation results, we find that $E_{F}$ often deviates by several millielectron volts from the charge-neutral point estimated by the calculations. Different samples of the same compound also have a difference of few milli-electron volts in their chemical potentials. Considering the narrow range of the Weyl cones in energy dispersion, these differences can result in a significant change in the FS topology.

We can tell the difference between the band structures for TaAs and the other three siblings after the QO measurements, although the samples differ significantly in their chemical potentials. The QO frequencies for the crystals of TaAs are generally smaller than those for the other three compounds when $B / / c$. An angular dependence study of the QOs suggested that the FS of TaAs has two types of electron pockets surrounding isolated Weyl nodes and one type of trivial hole pockets [73]. This result is consistent with the band structure calculations, which suggested that the energy difference between W1 and $\mathrm{W} 2$ is less than $20 \mathrm{meV}$ for TaAs. The small energy difference and the adaptable chemical potential promise that the carriers in TaAs at $E_{F}$ are Weyl quasiparticles with confirmed chirality [27]. 
Table 3 Summary of the QOs for the single crystals of the $\mathrm{Nb}(\mathrm{Ta}) \mathrm{P}(\mathrm{As})$ compounds. $F(T)$ is the frequency; $m_{c y c}$ is the cyclotron mass in units of the free electron mass; the intercept was obtained from the fitting of the Landau fan diagram and $\Phi_{B}$ is the Berry phase.

\begin{tabular}{|c|c|c|c|c|c|}
\hline Compound & $F / T\left(m_{c y c} / m_{e}\right)$ & Intercept & $\Phi_{B}$ & note & Ref. \\
\hline \multirow{5}{*}{$\mathrm{NbP}$} & $7,13,32(0.076)$ & - & - & $\mathrm{SdH}, H / / c$ & {$[25]$} \\
\hline & 15.3 & - & - & $\mathrm{SdH}, H / / c$ & {$[46]$} \\
\hline & $6.8,13.9(0.47), 31.8,64.4$ & 0.3 & - & $\mathrm{SdH}, H / / c$ & {$[59]$} \\
\hline & $8.7(0.05), 14.6(0.06), 32.1(0.05), 39.6(0.05)$ & - & - & $\mathrm{dHvA}, H / / c$ & {$[58]$} \\
\hline & $97(0.3), 324(0.7)$ & - & - & $\mathrm{dHvA}, H / / a$ & {$[58]$} \\
\hline \multirow{6}{*}{$\mathrm{TaP}$} & $25.6,49.6$ & -0.3958 & - & $\mathrm{SdH}, H / / c$ & {$[60]$} \\
\hline & $18(0.04), 44(0.08)$ & - & $0.91 \pi(18 \mathrm{~T}), 0.1 \pi(44 \mathrm{~T})$ & $\mathrm{SdH}, H / / c$ & {$[45]$} \\
\hline & $110(0.26), 150(0.24)$ & - & $0.91 \pi(110 \mathrm{~T}), 0.93 \pi(150 \mathrm{~T})$ & $\mathrm{SdH}, H / / a$ & {$[45]$} \\
\hline & 18.2 & - & - & $\mathrm{SdH}, H / / c$ & {$[46]$} \\
\hline & $15(0.021), 18(0.05), 25,51(0.11)$ & - & - & $\mathrm{SdH}$ and dHvA $H / / c$ & {$[47]$} \\
\hline & $\begin{array}{l}26(0.10), 34(0.13), 105(0.35), 295(1.24), \\
\quad 148(0.4)\end{array}$ & - & - & $\mathrm{SdH}$ and $\mathrm{dHvA}, H / / a$ & {$[47]$} \\
\hline \multirow{4}{*}{$\mathrm{NbAs}$} & 15.6(0.033), 20.8(0.066) & $0.12,0.36$ & $\pi, 0$ & $\mathrm{SdH}, H / / c$ & {$[62]$} \\
\hline & 101.5 and $106.2(0.046), 94$ and $97(0.029)$ & $0.35,0.12$ & $0, \pi$ & $\mathrm{SdH}, H / / a$ & {$[62]$} \\
\hline & 25 & 0.119 & $\pi$ & $\mathrm{SdH}, H / / c$ & {$[63]$} \\
\hline & $17.7,20.1$ & - & - & $\mathrm{dHvA}, H / / c$ & {$[75]$} \\
\hline \multirow{2}{*}{ TaAs } & $7.42-12.48(0.15)$ & 0 & - & $\mathrm{SdH}, H / / c$ & {$[27]$} \\
\hline & 7,16 & - & - & $\mathrm{SdH}, H / / c$ & {$[26]$} \\
\hline
\end{tabular}

To the contrary, band structure calculations suggested that the energy differences between the two types of Weyl nodes are approximately $50 \mathrm{meV}$ or more for $\mathrm{NbP}, \mathrm{NbAs}$, and TaP. This large energy difference indicates that at least one type of Weyl nodes is surrounded in pair by large electron or hole pockets, wherever the chemical potential is located. This assumption is consistent with the observed large QO frequencies in more than $20 \mathrm{~T}$ for these three compounds when $B / / c$ (Table 3 ).

An angular dependent QO measurements detected that the FS for NbP has a pair of spin-orbit-split electron pockets and a similar pair of hole pockets at $E_{F}$, in which one pocket nests inside the other for both electrons and holes [58]. The W1 nodes are fully merged into the electron pockets and lose their chirality, while the Weyl chirality of the W2 nodes remain intact in this situation. According to the QO measurements for a single crystal of TaP, the $\mathrm{W} 1$ and $\mathrm{W} 2$ points are all included in the banana-like-shaped electron and Hall pockets, respectively [47]. The chemical potentials are above 50
meV away from the $\mathrm{W} 1$ nodes, which denies their chirality.

Some critical parameters of the electron and hole pockets, such as the cyclotron mass $\left(m_{c y c}\right)$ and the Dingle temperature $\left(T_{D}\right)$, can be determined by the analyses of the thermal damping effect of the QOs [68]. The SdH oscillations of $\rho_{x x}$ for a 3D electron system [72] obey the following expression at a finite temperature:

$$
\rho_{x x}=\rho_{0}[1+A(B, T) \cos 2 \pi(F / B+\gamma)],
$$

where $\rho_{0}$ is the non-oscillatory part of the resistivity, $A(B, T)$ is the amplitude of the $\mathrm{SdH}$ oscillations, $B$ is the magnetic field, and $\gamma$ is the Onsager phase. The amplitude of the SdH oscillations can be fitted using the following Lifshitz-Kosevich formula for a 3D system [72]:

$$
A(B, T) \propto \exp \left(-2 \pi^{2} k_{B} T_{D} /\left(\hbar \omega_{c}\right)\right) \frac{2 \pi^{2} k_{B} T /\left(\hbar \omega_{c}\right)}{\sinh \left(2 \pi^{2} k_{B} T /\left(\hbar \omega_{c}\right)\right)}
$$


where $k_{B}$ is Boltzmann's constant and the cyclotron frequency is $\omega_{c}=e B / m_{c y c}$. The values of $m_{c y c}$ at different frequencies summarized in Table 3 were compared with the calculation results. Quantum mobility can be estimated from $T_{D}$ and $m_{c y c}$ as well. The quantum mobility was estimated as larger than $1 \times 10^{4} \mathrm{~cm}^{2} / \mathrm{Vs}$ in general, which is consistent with the Hall effect measurements [73].

A focal point of recent QO studies is the Onsager phase $\gamma$, which comes from the Berry phase of the electron pockets [74]. A band with linear energy dispersion carries an extra $\pi$ Berry phase in contract with a zero Berry phase for a topologically trivial parabolic dispersive band [69]. An additional phase shift of $\delta= \pm 1 / 8$ is dependent on the curvature counting the FS topology for a 3D electron system [68]. In theory, the combined phase shift should be the discrete values of $\gamma= \pm 1 / 8$ for Dirac and Weyl semimetals. In the data analyses, the resistivity peaks and valleys were used to identify the Landau indices $(n)$ and a plot of $n$ versus $1 / B$, called the Landau fan diagram, was used to extrapolate the phase shift $(\gamma)$. Summarizing the results for $\mathrm{Nb}(\mathrm{Ta}) \mathrm{As}(\mathrm{P})$ in Table 3, we realized that there are still many arguments to be resolved regarding the feasible data analyses in the determination. A detailed discussion of the determination of the Berry phase for Dirac and Weyl semimetals from their SdH oscillations can be found in Ref. [74].

\subsection{2 $\mathrm{Nb}(\mathrm{Ta}) \mathrm{As}(\mathrm{Sb})_{2}$}

All the electrical transport experiments on the $\mathrm{Nb}(\mathrm{Ta}) \mathrm{As}(\mathrm{Sb})_{2}$ compounds detected strong $\mathrm{SdH}$ oscillations at low temperatures, but the analyses of the angular dependence to map out the FS have rarely been done, except for that in Ref. [34], in which Shen et al. determined the frequencies of the $\mathrm{SdH}$ oscillations for $\mathrm{NbAs}_{2}$ when the field is tilted away from the $a b$ plane, and found that the frequency changes agree well with the band structure calculations [34]. The frequencies and $m_{c y c}$ of $\mathrm{Nb}(\mathrm{Ta}) \mathrm{As}(\mathrm{Sb})_{2}$ are summarized in Table 4 when the magnetic field is parallel and perpendicular to the $a b$ plane. The QO frequencies of $\mathrm{Nb}(\mathrm{Ta}) \mathrm{As}(\mathrm{Sb})_{2}$ are in general one order of magnitude larger than those for $\mathrm{Nb}(\mathrm{Ta}) \mathrm{P}(\mathrm{As})$. This result is consistent with the band structural calculations, which indicate that the FS of the $\mathrm{Nb}(\mathrm{Ta}) \mathrm{As}(\mathrm{Sb})_{2}$ compounds covers a larger area in their BZ $[34,56]$. The Onsager phase of the large frequencies for $\mathrm{Nb}(\mathrm{Ta}) \mathrm{As}(\mathrm{Sb})_{2}$ is difficult to determine.

\subsection{Negative longitudinal magnetoresistance}

One of the crucial signatures of the Weyl quasiparticles in electrical transport is the so-called chiral anomaly. The chiral anomaly in quantum field theory presents as the breaking of the chiral-charge conservation law, which is crucial in understanding a number of important aspects of the standard model in particle physics, such as the triangle anomaly associated with the decay of the neutral pion $\pi^{0}[76,77]$. The discovery of the Weyl semimetals provides the opportunity to test this exotic concept in table-top experiments. A signature of the chiral anomaly in electrical transport is the Adler-Bell-Jachiw (ABJ) anomaly, which presents a negative MR when the magnetic field and the current are parallel [78]. A positive MR occurs in regular metal in general, because the magnetic field shortens the electron mean-free path. In a Weyl semimetal, alongside the scattering effect, parallel magnetic and electric fields can pump the electrons between the Weyl nodes of opposite chirality, which are separated in momentum space. This charge pumping process violates the chiral-charge conservation law and is able to induce a negative MR instead. However, other mechanisms, such as scattering effects or even a currentjetting effect, can induce a negative LMR as well. The origin of the recently observed negative LMR in various Dirac and Weyl semimetals is still under debate (see

Table 4 Summary of the QOs for the single crystals of the $\mathrm{Nb}(\mathrm{Ta}) \mathrm{Pn}_{2}$ compounds.

\begin{tabular}{cccccc}
\hline Compound & $F / T\left(m_{\text {cyc }} / m_{e}\right)$ & Intercept & $\Phi_{B}$ & Note & Ref. \\
\hline \multirow{3}{*}{ NbAs $_{2}$} & $124(0.24), 224(0.21)$ & - & - & $\mathrm{SdH}, H \perp a b$ plane & {$[35]$} \\
\cline { 2 - 6 } & $122(0.24), 226(0.29), 76(0.21)$ & - & - & $\mathrm{SdH}, H \perp a b$ plane & {$[34]$} \\
\cline { 2 - 6 } & $204(0.27), 90(0.20)$ & 0 & - & $\mathrm{SdH}, H \perp a b$ plane & {$[66]$} \\
\hline \multirow{2}{*}{$\mathrm{TaAs}_{2}$} & $39(0.08), 54(0.14)$ & - & - & $\mathrm{SdH}, H \perp a b$ plane & {$[35]$} \\
\cline { 2 - 6 } & $45(0.17), 158(0.24)$ & 0 & - & $\mathrm{SdH}, H \perp a b$ & {$[66]$} \\
\hline$n$ & $190.5(0.34), 212.5(0.30)$ & - & - & $\mathrm{SdH}, H \perp a b$ & {$[33]$} \\
\hline $\mathrm{NbSb}_{2}$ & $227(0.68), 483(1.69)$ & - & - & $\mathrm{SdH}, H \perp a b$ & {$[32]$} \\
\hline $\mathrm{TaSb}_{2}$ & 220,465 & $0.29,0.2$ & - & $\mathrm{SdH}, H \perp a b$ & {$[36]$} \\
\hline
\end{tabular}


Ref. [79] and references therein). We notice contradictory conclusions from different experiments on the NbAsand the $\mathrm{OsGe}_{2}$-type compounds.

Figures 8(a) and (b) show representative features of negative LMR for the NbAs-type compounds at low temperatures. The LMR for $B / / i$ in TaAs shows a small dip in low magnetic field and then a negative signal in a moderate field following a re-entry to positive in higher field [26]. The negative part of the MR, which only occurs below $100 \mathrm{~K}$, is highly sensitive to small deviations of the directions of the field and current $[26,27]$. The small dip in low magnetic field was ascribed to a 3D weak anti-localization (WAL) effect. The re-entry to positive in higher field was ascribed to a slightly imperfect alignment between the field and the current in the experiments [26], or a scattering effect by a strong magnetic field [80]. On the other hand, the negative part of the LMR corresponding to a $B^{2}$-dependent magnetoconductance is consistent with a theoretically predicted power law of the chiral anomaly in weak field [81]. Figures $8(a)$ and (b) show that the negative LMR occurs for the two main crystallographic orientations $B / / i / / a$ and $B / / i / / c$, which is consistent with the feature that the ABJ anomaly does not rely on any specific orientation in type- $I$ WSM. Zhang et al. found that the negative
LMR is dependent on the chemical potentials in different samples: it is large in the samples with $E_{F}$ close to W2 but vanishes in the samples with $E_{F}$ away from W2 [27]. This dependence on the $E_{F}$ is expected from the dependence of the Berry curvature on chemical potential in the simplest model of a Weyl semimetal [Fig. 8(c)]. Negative LMR was observed in NbP, NbAs, and TaP as well [45, 59, 63]; however, other works in the literature reported small positive LMR on the same compounds $[25,46,61]$. On the other hand, the mechanism of the negative LMR for single crystals of TaP is in dispute. Arnold et al. claimed that the FS forms electron and hole pockets surrounding pairs of Weyl nodes in TaP [47], which means that the chirality is ill-defined in TaP. They attributed the negative LMR to a magnetic-fieldinduced current-jetting effect.

The measurement results on the LMR for the $\mathrm{Nb}(\mathrm{Ta}) \mathrm{As}(\mathrm{Sb})_{2}$ compounds is contradictory in the literature as well. Luo et al. reported that the LMR of $\mathrm{TaAs}_{2}$ reaches an extremely large number $(-98 \%)$ in 3 $\mathrm{T}$ at $2 \mathrm{~K}$, which persists up to $150 \mathrm{~K}$ [57] [Fig. 8(d)]. The longitudinal magneto-conductance can be well-fitted by a $B^{2}$ term, which behaves similarly to the ABJ-type chiral conductance. The reported negative LMR of $\mathrm{NbAs}_{2}$ and $\mathrm{TaSb}_{2}$ has a similar profile to that of TaP at low temper-
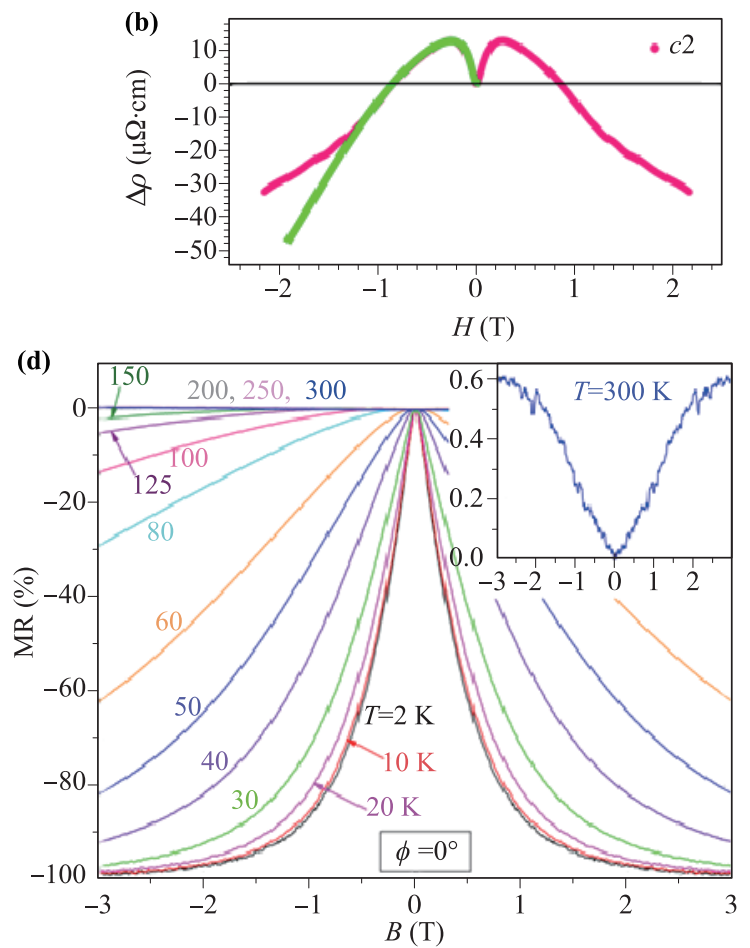

Fig. 8 (a, b) Negative LMR for samples of TaAs with different chemical potentials. The red lines are the data and the green lines are the theoretical fits. The magnetic field and the current are along $a$ axis in figure (a) but along $c$ axis in figure (b). (c) Dependence of the chiral coefficient $C_{W}$ normalized to other coefficients on chemical potential $\left(E_{F}\right)$. The observed scaling behavior is $1 / E_{F}^{2}$. Figures (a), (b) and (c) are reproduced from Ref. [27]. (d) Observed negative LMR of TaAs 2 at different temperatures (Reproduced from Ref. [57]). The inset shows the data at $300 \mathrm{~K}$. 
atures $[34,36]$. To the contrary, other measurements of $\mathrm{TaAs}_{2}, \mathrm{NbAs}_{2}$, and $\mathrm{NbSb}_{2}$ yielded observations of moderate, positive LMR with no sign of negative contributions $[32,33,35]$. All the conflicting results and explanations for the LMR for these two families of semimetals indicate that further elaboration is needed to clarify the origin of the negative LMR.

\subsection{Measurements in extreme conditions}

Pressure can compact or even change a material's crystal structure and tune its physical properties. The crystal structures of $\mathrm{NbP}$ and $\mathrm{NbAs}$ are quite robust under pressure $[51,82]$. The change in the SdH oscillations of NbAs under hydrostatic pressure reveals that its FS undergoes an anisotropic evolution but the topological features of the two observed pockets remain intact up to $2.3 \mathrm{GPa}$ [83]. In contrast, the structural and electrical properties of TaAs and TaP seem to be more easily affected by pressure. It was reported that TaAs undergoes a transition to a $\mathrm{P}-6 \mathrm{~m}_{2}$ structure at $14 \mathrm{GPa}$. Band structure calculations showed that this hexagonal TaAs is another Weyl semimetal possessing 12 Weyl nodes at an isoenergy level [84].

The existence of a superconducting ( $\mathrm{SC}$ ) phase under higher pressure is a pronounced feature in TaAs and TaP. The point-contact measurements found an SC phase with the on-site critical temperature of approximately $6 \mathrm{~K}$ at the interface between the tip and the TaAs single crystals, which is induced by the pressure applied by the tip [85]. The resistance measurements of TaP under the pressure of $70 \mathrm{GPa}$ also showed evidence of an SC transition, which became more prominent when the pressure was released to ambient [86]. First principle calculations suggest that $\mathrm{TaP}$ undergoes a structural transition at $70 \mathrm{GPa}$ to an orthorhombic phase, which may host this superconductivity.

The other extreme condition is an intense magnetic field, which can significantly influence the motion of the electrons in a metal, especially when it is beyond the QL. The QL is defined as the lowest Landau subband in which all the electrons are confined by a strong magnetic field while all other Landau subbands have successively passed through the chemical potential. In a $3 \mathrm{D}$ electron system, the Landau subbands acquire a dispersion that depends on the band structure, in the direction parallel to the applied field. The electron pockets with the small extremal cross-sectional areas for $H / / c$ in the NbAs-type compounds promise an accessible QL of Weyl semimetals in laboratory-based magnets. Moll et al. reported that the magnetic torque of $\mathrm{NbAs}$ is negative at low field but positive at high field, with a turn in slope at the QL [75]. The positive torque signal indicates that the system becomes paramagnetic above the QL, in stark contrast to its status as Landau diamagnetic below the QL. This unusual paramagnetic signal was ascribed to the field-independent energy of the zeroth Landau subband and the $\pi$ Berry phase of the Weyl electron pockets. Apart from the torque measurements, a sharp sign reversal of the Hall signal was observed in $\mathrm{TaP}$ in $33 \mathrm{~T}$ at low temperatures [64]. For the other NbAs-type compounds, the anomalies of the resistance and Hall signals are much weaker and only occur at very low temperatures. These anomalous features of the electrical transport signals may indicate a Fermi surface instability in strong field [87].

\section{$5 \quad$ Discussion and outlook}

Our survey on the transport properties of these two semimetallic families boils down to a crucial question: what is the unique transport property of Weyl quasipar-
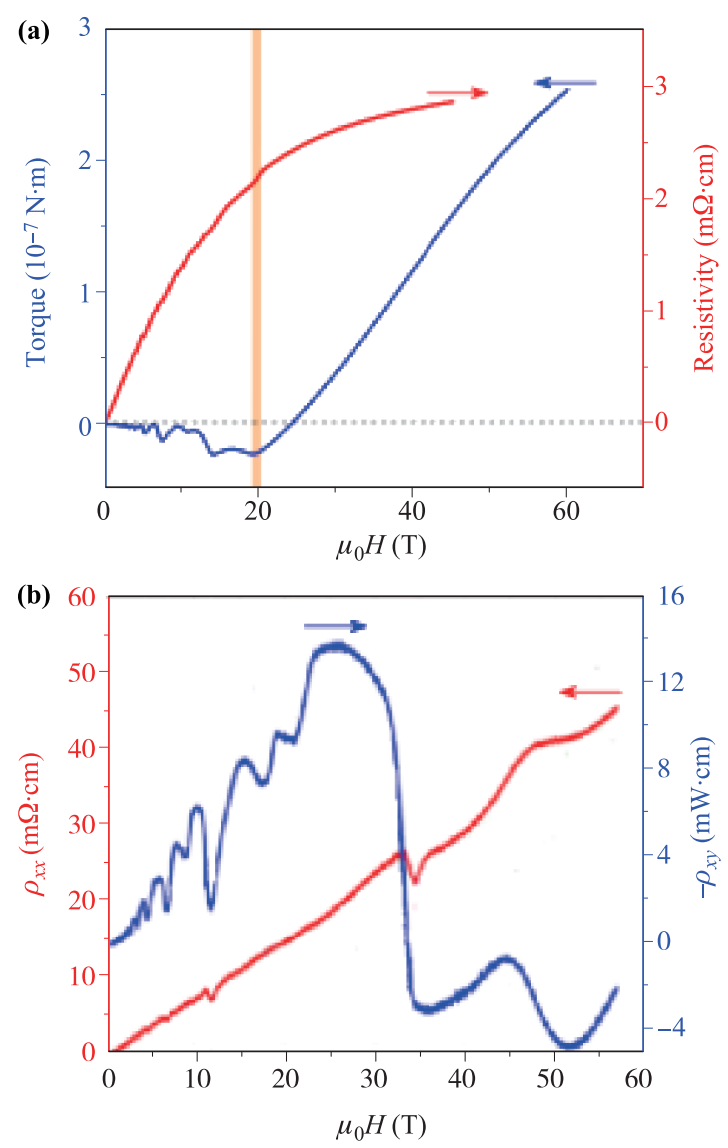

Fig. 9 (a) Magnetic torque and resistivity of NbAs across the quantum limit (QL) (Reproduced from Ref. [75]). Above the QL, the torque grows strongly in magnitude. (b) The resistivity and Hall resistivity of $\mathrm{TaP}$ in an intense magnetic field at $1.5 \mathrm{~K}$ and $4.2 \mathrm{~K}$ (Reproduced from Ref. [64]). 
ticles? Below, we inspect all the noteworthy transport properties one by one. After comparing the transversal MR for NbAs-type and $\mathrm{OsGe}_{2}$-type semimetals, we can conclude that a large MR at low temperatures is a very common feature in semimetals. Recent studies on single crystals, including semimetallic $\mathrm{WTe}_{2}[67,88]$, $\mathrm{Cd}_{3} \mathrm{As}_{2}$ [89], $\mathrm{PtSn}_{4}$ [90], and $\mathrm{PdCoO}_{2}$ [91], reported comparably large MR. Remember the MR for the prototype semimetal bismuth was reported to be as large as $1.6 \times 10^{7}$ at $4.2 \mathrm{~K}$ in $5 \mathrm{~T}$ [92], which is at least two orders of magnitude greater than the MRs for these compounds. Whatever the underlying mechanism, a large MR at low temperatures is very common for high-quality single crystals of semimetals.

The power law of the field-dependent MR for the topological semimetals has been recently noticed. A parabolic field-dependent MR is predicted in semimetals by a twoband model. In this model, when the electrons and holes are close to being compensated, a resonant effect will strongly enhance a parabolic MR to be unsaturated. This effect was believed to occur in bismuth, graphite, and $\mathrm{WTe}_{2}$ [67]. The Hall effect measurements and band structure calculations in $\mathrm{Nb}(\mathrm{Ta}) \mathrm{As}(\mathrm{Sb})_{2}$ revealed that the mechanism of their large parabolic MR should be the same. In contrast, the mechanism of a linear fielddependent MR observed in other semimetals is less understood. Abrikosov deduced that a quantum linear MR occurs near the crossing point of the conduction and valence bands with linear energy dispersion when the magnetic field is beyond the QL $[93,94]$. Abrikosov's prediction seems to be the same as the linearly dependent MR of NbP above the QL in Ref. [25]. However, this mechanism cannot explain the linear MR occurring in much lower field than the QL for other samples. Parish and Littiewood (PL) put forward another explanation for the linear MR based on the spatial mobility fluctuation [95]. The correlation between the electron mobility and linear MR indicates that the classical PL effect is plausible for the linear MR of TaP below the QL [46]. Another mechanism of the linear MR in the Weyl semimetals was also considered [96]. However, the survey tells us that the power law of the MR for $\mathrm{Nb}(\mathrm{Ta}) \mathrm{P}(\mathrm{As})$ is indeed dependent on the temperature, the strength of the magnetic field, and the particular samples. This intricacy seems to make any single mechanism of the linear MR unconvincing for $\mathrm{Nb}(\mathrm{Ta}) \mathrm{P}(\mathrm{As})$ at this point.

Another pronounced property for the NbAs-type compounds is their high electron mobilities on the order of $10^{5} \mathrm{~cm}^{2} /$ Vs. A recent theoretical work ascribed the high mobilities of the Weyl quasiparticles to the underlying protection mechanism against backscattering [97]. Our survey shows that the mobilities of the NbAs-type compounds are indeed one order of magnitude higher than those of the $\mathrm{Nb}(\mathrm{Ta}) \mathrm{As}(\mathrm{Sb})_{2}$ compounds in gen- eral. Recent works on various TaAs samples revealed that the mobilities are strongly enhanced in the samples whose $E_{F}$ is close to the Weyl nodes. We notice that the high-quality crystals of trivial semimetals, such as $\mathrm{Nb}(\mathrm{Ta}) \mathrm{As}(\mathrm{Sb})_{2}$, can manifest moderately high mobilities despite no topological protection mechanism.

Recent experimental works commonly adapted the determined QOs phase shift to clarify the $\pi$ Berry phase in topological semimetals. However, there are still some arguments about the correct way to extract the phase shift from the resistivity data in 3D systems. Very recently, Wang et al. clarified that the resistivity peaks correspond to the band edges of the Landau subbands and the integer Landau indices [74]. They also found that the phase shift is not a uniform number in data analyses when the chemical potential is near the Lifshitz transition point. There also exist some difficulties in detecting the phase shift by extrapolating the Landau fan diagram. When the magnetic field is not strong enough to access the low Landau subbands connected with large FS, determining the intercept in the fan diagram will introduce large uncertainty. The magnetic-field-induced Zeeman effect may also affect the Landau subband separation. Considering all the difficulties, we suggest that the phase shift in the QOs should be a signature but not explicit evidence of the topological character.

As mentioned above, recent studies ascribed the negative LMR to multiple possible mechanisms in various semimetals. The chiral anomaly is a popular explanation but the negative LMR can also occur through a purely classical geometric current-jetting effect in materials with high mobilities [47], or be caused by a scattering effect in a strong magnetic field beyond the QL $[98,99]$. On the other hand, the negative MR due to the chiral anomaly is a Berry curvature effect. A generic band structure without Weyl nodes can also carry nonzero Berry curvature, as long as the time-reversal symmetry or inversion symmetry is broken. It is not yet understood whether such a generic band structure with non-zero Berry curvature can induce a negative LMR. We notice that the negative LMR of TaAs shows a dependence on the chemical potential with respect to the energy of the Weyl nodes, which systematically tunes the Berry curvature field. This dependence constitutes a strong signature of the topological Weyl fermion physics. Nevertheless, a negative LMR should not be treated as a smoking gun of the existence of the Weyl quasiparticles.

Our survey underlines the importance of the singlecrystalline forms of compounds for physical measurements. All the fundamental properties of the Weyl semimetals, including the Weyl quasiparticles, topological Fermi arcs, and the chiral anomaly, are based on ARPES and magneto-transport experiments on single crystals. Apart from the bulk materials, new forms 
of the Weyl semimetals have been requested for the potential applications in prospective devices. For instance, a theoretical proposal predicted that the chiral charge pumping effect could lead to a dissipationless axial current in a long thin ribbon of a mesoscopic Weyl semimetal material [100]. A new type of QO that arises from the topological Fermi arc surfaces state is also expected to be observed in thin films of Weyl semimetals $[101,102]$. Furthermore, achieving the optical control of the Weyl physics has been proposed in a number of works in the literature [31]. All these developments give us motivation to re-examine the physical properties of the semimetal materials in their single-crystalline, mesoscopic-crystalline, and even thin-film forms. These experimental achievements and theoretical proposals indicate that the appropriate forms of the materials are crucial to attain deep understanding their topological properties.

Acknowledgements S. Jia was supported by the National Basic Research Program of China (Grant Nos. 2013CB921901 and 2014CB239302).

Open Access This article is distributed under the terms of the Creative Commons Attribution License which permits any use, distribution, and reproduction in any medium, provided the original author(s) and the source are credited.

\section{References}

1. D. Johrendt, C. Hieke, and T. Stürzer, 2.05 - transitionmetal pnictides, in: Comprehensive Inorganic Chemistry $\{\mathrm{II}\}$ (Second Edition), edited by Jan ReedijkKenneth Poeppelmeier, Amsterdam: Elsevier, 2013, pp. $111-135$

2. N. Schönberg, W. G. Overend, A. Munthe-Kaas, and N. A. Sörensen, An X-ray investigation of transition metal phosphides, Acta Chem. Scand. 8, 226 (1954)

3. H. Boller and E. Parthé, The transposition structure of NbAs and of similar monophosphides and arsenides of niobium and tantalum, Acta Crystallogr. 16(11), 1095 (1963)

4. S. Furuseth and A. Kjekshus, The crystal structure of NbAs (comments), Acta Crystallogr. 17(8), 1077 (1964)

5. S. Furuseth, A. Kjekshus, S. Åsen, H. Halvarson, and L. Nilsson, 0, On the arsenides and antimonides of niobium, Acta Chem. Scand. 18, 1180 (1964)

6. S. Furuseth, K. Selte, A. Kjekshus, S. Gronowitz, R. A. Hoffman, and A. Westerdahl, On the arsenides and antimonides of tantalum, Acta Chem. Scand. 19, 95 (1965)

7. J. J. Murray, J. B. Taylor, L. D. Calvert, Y. Wang, E. J. Gabe, and J. G. Despault, Phase relationships and thermodynamics of refractory metal pnictides: The metal-rich tantalum arsenides, J. Less Common Met. 46(2), 311 (1976)

8. S.-M. Huang, S.-Y. Xu, I. Belopolski, C.-C. Lee, G. Chang, B. K. Wang, N. Alidoust, G. Bian, M. Neupane, C. Zhang, S. Jia, A. Bansil, H. Lin, and M. Z. Hasan, A Weyl fermion semimetal with surface Fermi arcs in the transition metal monopnictide TaAs class, Nat. Commun. 6, 7373 (2015)

9. H. Weng, C. Fang, Z. Fang, B. A. Bernevig, and $\mathrm{X}$. Dai, Weyl semimetal phase in noncentrosymmetric transition-metal monophosphides, Phys. Rev. X 5(1), 011029 (2015)

10. F. Hulliger, New representatives of the $\mathrm{NbAs}_{2}$ and $\mathrm{ZrAs}_{2}$ structures, Nature 204(4960), 775 (1964)

11. W. Bensch and W. Heid, $\mathrm{NbAs}_{2}$, Acta Crystallogr. C 51(11), 2205 (1995)

12. G. S. Saini, L. D. Calvert, and J. B. Taylor, Preparation and characterization of crystals of MX- and $\mathrm{MX}_{2}$ type arsenides of niobium and tantalum, Can. J. Chem. 42(3), 630 (1964)

13. R. Nesper, The Zintl-Klemm concept - A historical survey, Zeitschrift für anorganische und allgemeine Chemie 640, 2639 (2014)

14. P. Alemany and S. Alvarez, Theoretical study of bonding and electrical conductivity in compounds with the $\mathrm{NbAs}_{2}$ structure, Inorg. Chem. 31(14), 3007 (1992)

15. J. Xu, M. Greenblatt, T. Emge, P. Hohn, T. Hughbanks, and Y. Tian, Crystal structure, electrical transport, and magnetic properties of niobium monophosphide, Inorg. Chem. 35(4), 845 (1996)

16. B. Saparov, J. E. Mitchell, and A. S. Sefat, Properties of binary transition-metal arsenides (TAs), Supercond. Sci. Technol. 25(8), 084016 (2012)

17. F. Failamani, P. Broz, D. Macciò, S. Puchegger, H. Müller, L. Salamakha, H. Michor, A. Grytsiv, A. Saccone, E. Bauer, G. Giester, and P. Rogl, Constitution of the systems $\{\mathrm{V}, \mathrm{Nb}, \mathrm{Ta}\} \mathrm{Sb}$ and physical properties of di-antimonides $\{\mathrm{V}, \mathrm{Nb}, \mathrm{Ta}\} \mathrm{Sb}_{2}$, Intermetallics 65, 94 (2015)

18. S. Y. Xu, I. Belopolski, N. Alidoust, M. Neupane, G. Bian, C. Zhang, R. Sankar, G. Chang, Z. Yuan, C. C. Lee, S.M. Huang, H. Zheng, J. Ma, D. S. Sanchez, B. Wang, A. Bansil, F. Chou, P. P. Shibayev, H. Lin, S. Jia, and M. Z. Hasan, Discovery of a Weyl fermion semimetal and topological Fermi arcs, Science 349(6248), 613 (2015)

19. B. Q. Lv, H. M. Weng, B. B. Fu, X. P. Wang, H. Miao, J. Ma, P. Richard, X. C. Huang, L. X. Zhao, G. F. Chen, Z. Fang, X. Dai, T. Qian, and H. Ding, Experimental discovery of Weyl semimetal TaAs, Phys. Rev. X 5(3), 031013 (2015)

20. L. X. Yang, Z. K. Liu, Y. Sun, H. Peng, H. F. Yang, T. Zhang, B. Zhou, Y. Zhang, Y. F. Guo, M. Rahn, D. Prabhakaran, Z. Hussain, S. K. Mo, C. Felser, B. Yan, and Y. L. Chen, Weyl semimetal phase in the noncentrosymmetric compound TaAs, Nat. Phys. 11(9), 728 (2015) 
21. S. Y. Xu, N. Alidoust, I. Belopolski, Z. Yuan, G. Bian, T. R. Chang, H. Zheng, V. N. Strocov, D. S. Sanchez, G. Chang, C. Zhang, D. Mou, Y. Wu, L. Huang, C. C. Lee, S. M. Huang, B. K. Wang, A. Bansil, H. T. Jeng, T. Neupert, A. Kaminski, H. Lin, S. Jia, and M. Z. Hasan, Discovery of a Weyl fermion state with Fermi arcs in niobium arsenide, Nat. Phys. 11(9), 748 (2015)

22. N. Xu, H. M. Weng, B. Q. Lv, C. E. Matt, J. Park, F. Bisti, V. N. Strocov, D. Gawryluk, E. Pomjakushina, K. Conder, N. C. Plumb, M. Radovic, G. Autès, O. V. Yazyev, Z. Fang, X. Dai, T. Qian, J. Mesot, H. Ding, and M. Shi, Observation of Weyl nodes and Fermi arcs in tantalum phosphide, Nat. Commun. 7, 11006 (2016)

23. S. Y. Xu, I. Belopolski, D. S. Sanchez, C. Zhang, G. Chang, C. Guo, G. Bian, Z. Yuan, H. Lu, T.-R. Chang, P. P. Shibayev, M. L. Prokopovych, N. Alidoust, H. Zheng, C.-C. Lee, S.-M. Huang, R. Sankar, F. Chou, C.-H. Hsu, H.-T. Jeng, A. Bansil, T. Neupert, V. N. Strocov, H. Lin, S. Jia, and M. Z. Hasan, Experimental discovery of a topological Weyl semimetal state in TaP, Sci. Adv. 1(10), e1501092 (2015)

24. C. L. Zhang, Z. J. Yuan, Q. D. Jiang, Z. Lin, B. B. Tong, X. X. Zhang, C. Xie, and S. Jia, Electron scattering in tantalum monoarsenide, Phys. Rev. B 95(8), 085202 (2017)

25. C. Shekhar, A. K. Nayak, Y. Sun, M. Schmidt, M. Nicklas, I. Leermakers, U. Zeitler, Y. Skourski, J. Wosnitza, Z. Liu, Y. Chen, W. Schnelle, H. Borrmann, Y. Grin, C. Felser, and B. Yan, Extremely large magnetoresistance and ultrahigh mobility in the topological Weyl semimetal candidate NbP, Nat. Phys. 10, 1038 (2015)

26. X. Huang, L. Zhao, Y. Long, P. Wang, D. Chen, Z. Yang, H. Liang, M. Xue, H. Weng, Z. Fang, X. Dai, and G. Chen, Observation of the chiral-anomaly-induced negative magnetoresistance in 3D Weyl semimetal TaAs, Phys. Rev. X 5(3), 031023 (2015)

27. C. L. Zhang, S. Y. Xu, I. Belopolski, Z. Yuan, Z. Lin, B. Tong, G. Bian, N. Alidoust, C. C. Lee, S. M. Huang, T. R. Chang, G. Chang, C. H. Hsu, H. T. Jeng, M. Neupane, D. S. Sanchez, H. Zheng, J. Wang, H. Lin, C. Zhang, H. Z. Lu, S. Q. Shen, T. Neupert, M. Z. Hasan, and S. Jia, Signatures of the Adler-Bell-Jackiw chiral anomaly in a Weyl fermion semimetal, Nat. Commun. 7, 10735 (2016)

28. H. Zheng, S.-Y. Xu, G. Bian, C. Guo, G. Chang, D. S. Sanchez, I. Belopolski, C.-C. Lee, S.-M. Huang, X. Zhang, R. Sankar, N. Alidoust, T.-R. Chang, F. Wu, T. Neupert, F. Chou, H.-T. Jeng, N. Yao, A. Bansil, S. Jia, H. Lin, and M. Z. Hasan, Atomic-scale visualization of quantum interference on a Weyl semimetal surface by Scanning Tunneling Microscopy, ACS Nano 10, 1378 (2016)

29. R. Batabyal, N. Morali, N. Avraham, Y. Sun, M. Schmidt, C. Felser, A. Stern, B. Yan, and H. Beidenkopf, Visualizing weakly bound surface Fermi arcs and their correspondence to bulk Weyl fermions, Sci. Adv. 2(8), e1600709 (2016)
30. H. Inoue, A. Gyenis, Z. Wang, J. Li, S. W. Oh, S. Jiang, N. Ni, B. A. Bernevig, and A. Yazdani, Quasiparticle interference of the Fermi arcs and surface-bulk connectivity of a Weyl semimetal, Science 351(6278), 1184 (2016)

31. S. Jia, S. Y. Xu, and M. Z. Hasan, Weyl semimetals, Fermi arcs and chiral anomalies, Nat. Mater. 15(11), 1140 (2016)

32. K. Wang, D. Graf, L. Li, L. Wang, and C. Petrovic, Anisotropic giant magnetoresistance in $\mathrm{NbSb}_{2}$, Sci. Rep. 4, 7328 (2014)

33. D. Wu, J. Liao, W. Yi, X. Wang, P. Li, H. Weng, Y. Shi, Y. Li, J. Luo, X. Dai, and Z. Fang, Giant semiclassical magnetoresistance in high mobility $\mathrm{TaAs}_{2}$ semimetal, Appl. Phys. Lett. 108, 042105 (2016)

34. B. Shen, X. Deng, G. Kotliar, and N. Ni, Fermi surface topology and negative longitudinal magnetoresistance observed in the semimetal $\mathrm{NbAs}_{2}$, Phys. Rev. B 93(19), 195119 (2016)

35. Z. Yuan, H. Lu, Y. Liu, J. Wang, and S. Jia, Large magnetoresistance in compensated semimetals $\mathrm{TaAs}_{2}$ and $\mathrm{NbAs}_{2}$, Phys. Rev. B 93(18), 184405 (2016)

36. Y. Li, L. Li, J. Wang, T. Wang, X. Xu, C. Xi, C. Cao, and J. Dai, Resistivity plateau and negative magnetoresistance in the topological semimetal $\mathrm{TaSb}_{2}$, Phys. Rev. B 94(12), 121115 (2016)

37. Y. Sun, S. C. Wu, and B. Yan, Topological surface states and Fermi arcs of the noncentrosymmetric Weyl semimetals TaAs, TaP, NbAs, and NbP, Phys. Rev. B 92(11), 115428 (2015)

38. M. Z. Hasan and C. L. Kane, Topological insulators, Rev. Mod. Phys. 82(4), 3045 (2010)

39. Z. Wang, Y. Sun, X. Q. Chen, C. Franchini, G. Xu, H. Weng, X. Dai, and Z. Fang, Dirac semimetal and topological phase transitions in $\mathrm{A}_{3} \mathrm{Bi}(\mathrm{A}=\mathrm{Na}, \mathrm{K}, \mathrm{Rb})$, Phys. Rev. B 85(19), 195320 (2012)

40. Z. Wang, H. Weng, Q. Wu, X. Dai, and Z. Fang, Threedimensional Dirac semimetal and quantum transport in $\mathrm{Cd}_{3} \mathrm{As}_{2}$, Phys. Rev. B 88(12), 125427 (2013)

41. X. Wan, A. M. Turner, A. Vishwanath, and S. Y. Savrasov, Topological semimetal and Fermi-arc surface states in the electronic structure of pyrochlore iridates, Phys. Rev. B 83(20), 205101 (2011)

42. G. Xu, H. Weng, Z. Wang, X. Dai, and Z. Fang, Chern semimetal and the quantized anomalous Hall effect in $\mathrm{HgCr}_{2} \mathrm{Se}_{4}$, Phys. Rev. Lett. 107(18), 186806 (2011)

43. P. Hosur and X. Qi, Recent developments in transport phenomena in Weyl semimetals, C. R. Phys. 14(9-10), 857 (2013)

44. J. J. Murray, J. B. Taylor, and L. Usner, Halogen transport of molybdenum arsenides and other transition metal pnictides, J. Cryst. Growth 15(3), 231 (1972)

45. J. Hu, J. Y. Liu, D. Graf, S. M. A. Radmanesh, D. J. Adams, A. Chuang, Y. Wang, I. Chiorescu, J. Wei, L. Spinu, and Z. Q. Mao, Berry phase and Zeeman splitting of Weyl semimetal TaP, Sci. Rep. 6(1), 18674 (2016) 
46. C. Zhang, C. Guo, H. Lu, X. Zhang, Z. Yuan, Z. Lin, J. Wang, and S. Jia, Large magnetoresistance over an extended temperature regime in monophosphides of tantalum and niobium, Phys. Rev. B 92(4), 041203 (2015)

47. F. Arnold, C. Shekhar, S. C. Wu, Y. Sun, R. D. dos Reis, N. Kumar, M. Naumann, M. O. Ajeesh, M. Schmidt, A. G. Grushin, J. H. Bardarson, M. Baenitz, D. Sokolov, H. Borrmann, M. Nicklas, C. Felser, E. Hassinger, and B. Yan, Negative magnetoresistance without well-defined chirality in the Weyl semimetal TaP, Nat. Commun. 7, 11615 (2016)

48. Z. Li, H. Chen, S. Jin, D. Gan, W. Wang, L. Guo, and X. Chen, Weyl semimetal TaAs: Crystal growth, morphology, and thermodynamics, Cryst. Growth Des. 16(3), 1172 (2016)

49. P. Schmidt, M. Binnewies, R. Glaum, and M. Schmidt, Chemical vapor transport reactions-methods, materials, modeling, in: Advanced Topics on crystal growth, InTech, 2013, Chap. 9

50. P. C. Canfield and Z Fisk, Growth of single crystals from metallic fluxes, Philos. Mag. B 65(6), 1117 (1992)

51. J. Zhang, F. L. Liu, J. K. Dong, X. Yang, N. N. Li, W. G. Yang, and S. Y. Li, Structural and transport properties of the Weyl semimetal NbAs at high pressure, Chin. Phys. Lett. 32(9), 097102 (2015)

52. T. Besara, D. A. Rhodes, K. W. Chen, S. Das, Q. R. Zhang, J. Sun, B. Zeng, Y. Xin, L. Balicas, R. E. Baumbach, E. Manousakis, D. J. Singh, and T. Siegrist, Coexistence of Weyl physics and planar defects in the semimetals TaP and TaAs, Phys. Rev. B 93(24), 245152 (2016)

53. J. O. Willerstrom, Stacking disorder in NbP, TaP, NbAs, and TaAs, J. Less Common Met. 99(2), 273 (1984)

54. C. C. Lee, S. Y. Xu, S. M. Huang, D. S. Sanchez, I. Belopolski, G. Chang, G. Bian, N. Alidoust, H. Zheng, M. Neupane, B. Wang, A. Bansil, M. Z. Hasan, and H. Lin, Fermi surface interconnectivity and topology in Weyl fermion semimetals TaAs, TaP, NbAs, and NbP, Phys. Rev. B 92(23), 235104 (2015)

55. Z. K. Liu, L. X. Yang, Y. Sun, T. Zhang, H. Peng, H. F. Yang, C. Chen, Y. Zhang, Y. F. Guo, D. Prabhakaran, M. Schmidt, Z. Hussain, S. K. Mo, C. Felser, B. Yan, and Y. L. Chen, Evolution of the Fermi surface of Weyl semimetals in the transition metal pnictide family, Nat. Mater. 15(1), 27 (2016)

56. C. Xu, J. Chen, G. X. Zhi, Y. Li, J. Dai, and C. Cao, Electronic structures of transition metal dipnictides $\mathrm{XPn}_{2}(\mathrm{X}=\mathrm{Ta}, \mathrm{Nb} ; \mathrm{Pn}=\mathrm{P}, \mathrm{As}, \mathrm{Sb})$, Phys. Rev. B 93(19), 195106 (2016)

57. Y. Luo, R. D. McDonald, P. F. S. Rosa, B. Scott, N. Wakeham, N. J. Ghimire, E. D. Bauer, J. D. Thompson, and F. Ronning, Anomalous electronic structure and magnetoresistance in $\mathrm{TaAs}_{2}$, Sci. Rep. 6(1), 27294 (2016)
58. J. Klotz, S. C. Wu, C. Shekhar, Y. Sun, M. Schmidt, M. Nicklas, M. Baenitz, M. Uhlarz, J. Wosnitza, C. Felser, and B. Yan, Quantum oscillations and the Fermi surface topology of the Weyl semimetal NbP, Phys. Rev. B 93(12), 121105 (2016)

59. Z. Wang, Y. Zheng, Z. Shen, Y. Lu, H. Fang, F. Sheng, Y. Zhou, X. Yang, Y. Li, C. Feng, and Z.-A. Xu, Helicity-protected ultrahigh mobility Weyl fermions in NbP, Phys. Rev. B 93, 121112 (2016)

60. J. Du, H. Wang, Q. Mao, R. Khan, B. Xu, Y. Zhou, Y. Zhang, J. Yang, B. Chen, C. Feng, and M. Fang, Large Unsaturated positive and negative magnetoresistance in Weyl Semimetal TaP, Sci. China Phys. Mech. Astron. $59(5), 657406$ (2015)

61. N. J. Ghimire, Y. Luo, M. Neupane, D. J. Williams, E. D. Bauer, and F. Ronning, Magnetotransport of single crystalline NbAs, J. Phys.: Condens. Matter 27(15), 152201 (2015)

62. Y. Luo, N. J. Ghimire, M. Wartenbe, H. Choi, M. Neupane, R. D. McDonald, E. D. Bauer, J. Zhu, J. D. Thompson, and F. Ronning, Electron-hole compensation effect between topologically trivial electrons and nontrivial holes in NbAs, Phys. Rev. B 92, 205134 (2015)

63. X. Yang, Y. Liu, Z. Wang, Y. Zheng, and Z. Xu, Chiral anomaly induced negative magnetoresistance in topological Weyl semimetal NbAs, arXiv: 1506.03190 (2015)

64. C. Zhang, Z. Lin, C. Guo, S. Y. Xu, C. C. Lee, H. Lu, S. M. Huang, G. Chang, C. H. Hsu, H. Lin, L. Li, C. Zhang, T. Neupert, M. Zahid Hasan, J. Wang, and S. Jia, Quantum phase transitions in Weyl semimetal tantalum monophosphide, arXiv: 1507.06301 (2015)

65. C. M. Hurd, in: The Hall Effect in Metals and Alloys, New York: Cambridge University Press, 1972

66. Y. Y. Wang, Q. H. Yu, P. J. Guo, K. Liu, and T. L. Xia, Resistivity plateau and extremely large magnetoresistance in $\mathrm{NbAs}_{2}$ and $\mathrm{TaAs}_{2}$, Phys. Rev. B 94(4), 041103 (2016)

67. M. N. Ali, J. Xiong, S. Flynn, J. Tao, Q. D. Gibson, L. M. Schoop, T. Liang, N. Haldolaarachchige, M. Hirschberger, N. P. Ong, and R. J. Cava, Large, nonsaturating magnetoresistance in $\mathrm{WTe}_{2}$, Nature 514, 205 (2014)

68. D. Shoenberg, Magnetic Oscillations in Metals, Cambridge University Press, 2009

69. G. P. Mikitik and Yu. V. Sharlai, Manifestation of Berry's phase in metal physics, Phys. Rev. Lett. 82(10), 2147 (1999)

70. G. P. Mikitik and Yu. V. Sharlai, Berry phase and de Haas-van Alphen effect in LaRhIn 5 , Phys. Rev. Lett. 93(10), 106403 (2004)

71. G. P. Mikitik and Yu. V. Sharlai, Berry phase and the phase of the Shubnikov-de Haas oscillations in threedimensional topological insulators, Phys. Rev. B 85(3), 033301 (2012) 
72. H. Murakawa, M. S. Bahramy, M. Tokunaga, Y. Kohama, C. Bell, Y. Kaneko, N. Nagaosa, H. Y. Hwang, and Y. Tokura, Detection of Berry's phase in a bulk Rashba semiconductor, Science 342(6165), 1490 (2013)

73. F. Arnold, M. Naumann, S. C. Wu, Y. Sun, M. Schmidt, H. Borrmann, C. Felser, B. Yan, and E. Hassinger, Chiral Weyl pockets and Fermi surface topology of the Weyl semimetal TaAs, Phys. Rev. Lett. 117(14), 146401 (2016)

74. C. M. Wang, H. Z. Lu, and S. Q. Shen, Anomalous phase shift of quantum oscillations in 3D topological semimetals, Phys. Rev. Lett. 117(7), 077201 (2016)

75. P. J. W. Moll, A. C. Potter, N. L. Nair, B. J. Ramshaw, K. A. Modic, S. Riggs, B. Zeng, N. J. Ghimire, E. D. Bauer, R. Kealhofer, F. Ronning, and J. G. Analytis, Magnetic torque anomaly in the quantum limit of Weyl semimetals, Nat. Commun. 7, 12492 (2016)

76. S. L. Adler, Axial-vector vertex in spinor electrodynamics, Phys. Rev. 177(5), 2426 (1969)

77. J. S. Bell and R. Jackiw, A PCAC puzzle: $\pi^{0} \rightarrow \gamma \gamma$ in the $\sigma$-model, Nuovo Cim., A 60(1), 47 (1969)

78. H. B. Nielsen and M. Ninomiya, The Adler-Bell-Jackiw anomaly and Weyl fermions in a crystal, Phys. Lett. B 130(6), 389 (1983)

79. X. T. Xu and S. Jia, Recent observations of negative longitudinal magnetoresistance in semimetal, Chin. Phys. B 25(11), 117204 (2016)

80. C. Z. Chen, H. Liu, H. Jiang, and X. C. Xie, Positive magnetoconductivity of Weyl semimetals in the ultraquantum limit, Phys. Rev. B 93(16), 165420 (2016)

81. D. T. Son and B. Z. Spivak, Chiral anomaly and classical negative magnetoresistance of Weyl metals, Phys. Rev. B 88(10), 104412 (2013)

82. R. D. dos Reis, S. C. Wu, Y. Sun, M. O. Ajeesh, C. Shekhar, M. Schmidt, C. Felser, B. Yan, and M. Nicklas, Pressure tuning the Fermi surface topology of the Weyl semimetal NbP, Phys. Rev. B 93(20), 205102 (2016)

83. Y. Luo, N. J. Ghimire, E. D. Bauer, J. D. Thompson, and F. Ronning, "Hard" crystalline lattice in the Weyl semimetal NbAs, J. Phys.: Condens. Matter 28(5), 055502 (2016)

84. Y. Zhou, P. Lu, Y. Du, X. Zhu, G. Zhang, R. Zhang, D. Shao, X. Chen, X. Wang, M. Tian, J. Sun, X. Wan, Z. Yang, W. Yang, Y. Zhang, and D. Xing, Pressureinduced new topological Weyl semimetal phase in TaAs, Phys. Rev. Lett. 117(14), 146402 (2016)

85. H. Wang, H. Wang, Y. Chen, J. Luo, Z. Yuan, J. Liu, Y. Wang, S. Jia, X.J. Liu, J. Wei, and J. Wang, Reply to Comment on Tip induced unconventional superconductivity on Weyl semimetal TaAs, arXiv: 1607.02886 (2016)

86. Y. Li, Y. Zhou, Z. Guo, X. Chen, P. Lu, X. Wang, C. An, Y. Zhou, J. Xing, G. Du, X. Zhu, H. Yang, J. Sun, Z. Yang, Y. Zhang, and H.H. Wen, Superconductivity induced by high pressure in Weyl semimetal TaP, arXiv: 1611.02548 [cond-mat.suprcon] (2016)
87. C. L. Zhang, B. Tong, Z. Yuan, Z. Lin, J. Wang, J. Zhang, C. Y. Xi, Z. Wang, S. Jia, and C. Zhang, Signature of chiral fermion instability in the Weyl semimetal TaAs above the quantum limit, Phys. Rev. B 94(20), 205120 (2016)

88. Z. Zhu, X. Lin, J. Liu, B. Fauque, Q. Tao, C. Yang, Y. Shi, and K. Behnia, Quantum oscillations, thermoelectric coefficients, and the Fermi surface of semimetallic $\mathrm{WTe}_{2}$, Phys. Rev. Lett. 114(17), 176601 (2015)

89. T. Liang, Q. Gibson, M. N. Ali, M. Liu, R. J. Cava, and N. P. Ong, Ultrahigh mobility and giant magnetoresistance in the Dirac semimetal $\mathrm{Cd}_{3} \mathrm{As}_{2}$, Nat. Mater. 14(3), $280(2014)$

90. E. Mun, H. Ko, G. J. Miller, G. D. Samolyuk, S. L. Bud'ko, and P. C. Canfield, Magnetic field effects on transport properties of $\mathrm{PtSn}_{4}$, Phys. Rev. B 85(3), 035135 (2012)

91. H. Takatsu, J. J. Ishikawa, S. Yonezawa, H. Yoshino, T. Shishidou, T. Oguchi, K. Murata, and Y. Maeno, Extremely large magnetoresistance in the nonmagnetic metal $\mathrm{PdCoO}_{2}$, Phys. Rev. Lett. 111(5), 056601 (2013)

92. P. B. Alers and R. T. Webber, The magnetoresistance of bismuth crystals at low temperatures, Phys. Rev. 91(5), 1060 (1953)

93. A. A. Abrikosov, Quantum magnetoresistance, Phys. Rev. B 58(5), 2788 (1998)

94. A. A. Abrikosov, Quantum linear magnetoresistance, Europhys. Lett. 49(6), 789 (2000)

95. M. M. Parish and P. B. Littlewood, Non-saturating magnetoresistance in heavily disordered semiconductors, Nature 426(6963), 162 (2003)

96. Y. Pan, H. Wang, P. Lu, J. Sun, B. Wang, and D.Y. Xing, The large unsaturated magnetoresistance of Weyl semimetals, arXiv: 1509.03975 [cond-mat.meshall] (2015)

97. Q. D. Jiang, H. Jiang, H. Liu, Q. F. Sun, and X. C. Xie, Chiral wave-packet scattering in Weyl semimetals, Phys. Rev. B 93(19), 195165 (2016)

98. P. N. Argyres and E. N. Adams, Longitudinal magnetoresistance in the quantum limit, Phys. Rev. 104, 900 (1956)

99. H. Z. Lu, S. B. Zhang, and S. Q. Shen, High-field magnetoconductivity of topological semimetals with shortrange potential, Phys. Rev. B 92(4), 045203 (2015)

100. S. A. Parameswaran, T. Grover, D. A. Abanin, D. A. Pesin, and A. Vishwanath, Probing the chiral anomaly with nonlocal transport in three-dimensional topological semimetals, Phys. Rev. X 4(3), 031035 (2014)

101. A. C. Potter, I. Kimchi, and A. Vishwanath, Quantum oscillations from surface Fermi arcs in Weyl and Dirac semimetals, Nat. Commun. 5, 5161 (2014)

102. P. J. W. Moll, N. L. Nair, T. Helm, A. C. Potter, I. Kimchi, A. Vishwanath, and J. G. Analytis, Transport evidence for Fermi-arc-mediated chirality transfer in the Dirac semimetal $\mathrm{Cd}_{3} \mathrm{As}_{2}$, Nature 535(7611), 266 (2016) 